\title{
On the probabilities of branch durations and stratigraphic gaps in phylogenies of fossil taxa when rates of diversification and sampling vary over time
}

\author{
Peter J. Wagner (D)
}

\begin{abstract}
The time separating the first appearances of species from their divergences from related taxa affects assessments of macroevolutionary hypotheses about rates of anatomical or ecological change. Branch durations necessarily posit stratigraphic gaps in sampling within a clade over which we have failed to sample predecessors (ancestors) and over which there are no divergences leading to sampled relatives (sister taxa). The former reflects only sampling rates, whereas the latter reflects sampling, origination, and extinction rates. Because all three rates vary over time, the probability of a branch duration of any particular length will differ depending on when in the Phanerozoic that branch duration spans. Here, I present a birth-death-sampling model allowing interval-to-interval variation in diversification and sampling rates. Increasing either origination or sampling rates increases the probability of finding sister taxa that diverge both during and before intervals of high sampling/origination. Conversely, elevated extinction reduces the probability of divergences from sampled sister taxa before and during intervals of elevated extinction. In the case of total extinction, a Signor-Lipps will reduce expected sister taxa leading up to the extinction, with the possible effect stretching back many millions of years when sampling is low. Simulations indicate that this approach provides reasonable estimates of branch duration probabilities under a variety of circumstances. Because current probability models for describing morphological evolution are less advanced than methods for inferring diversification and sampling rates, branch duration priors allowing for timevarying diversification could be a potent tool for phylogenetic inference with fossil data.
\end{abstract}

Peter J. Wagner. Department of Earth E Atmospheric Sciences and School of Biological Sciences. 316 Bessey Hall, University of Nebraska, Lincoln, Nebraska 68588, U.S.A. E-mail: pjwagner3@gmail.com. ORCID: 0000-00029083-9787

Accepted: 14 September 2018

Data available from the Dryad Digital Repository: https://datadryad.org/resource/doi:10.5061/dryad. 926bs5d

\section{Introduction}

The divergence times implicit to phylogenetic relationships among fossil taxa almost always posit durations over which we have failed to sample taxa from a clade and thus imply gaps in stratigraphic sampling (Smith 1988). Given the challenges that easily fossilized characters present to phylogenetic analyses, such as abundant homoplasy and correlated change (e.g., Wagner 2000a; Smith 2001b; Wagner and Estabrook 2015; Wright et al. 2016; Sansom and Wills 2017), the plausibility of the stratigraphic gaps implicit to branch durations is critical for evaluating the plausibility of general sets of relationships (Fisher 1994). Within the context of particular cladistic relationships, the probabilities of branch durations are important for assessing macroevolutionary hypotheses about consistency of rates of anatomical change and/or ecological change (Ruta et al. 2006;
Friedman 2010; Bapst et al. 2016; Halliday and Goswami 2016) and for accommodating the effects of rate variation and even character coding practices on methods that try to calibrate divergence times using local morphological "clocks" (i.e., tip-dating; Ronquist et al. 2012; Herrera and Dávalos 2016; Matzke and Wright 2016; Matzke and Irmis 2018).

The probabilities of branch durations and associated stratigraphic gaps reflect not just sampling rates, but also origination and extinction rates (e.g., Foote et al. 1999; Stadler 2010; Bapst 2013; Heath et al. 2014). Ideally, if we can put prior probabilities on divergence times given extrinsic information about these rates, then analyses using birth-deathsampling models to estimate divergence times and basic phylogenetic relationships among fossil taxa (e.g., Wright 2017; Wright and Toom 2017) will be more robust to local idiosyncrasies in rates or modes of morphological 
change. Marshall (2017) draws attention to the fact that existing birth-death-sampling models for estimating prior probabilities of phylogenetic branches do not take into account existing paleobiological estimates of rates of diversification or sampling. Although some models do allow for heterogeneity in these rates among taxa (e.g., Stadler et al. 2013, 2018), existing methods do not accommodate the clade-wide shifts in these rates from one interval to the next that the fossil record demonstrates. Currently, our methods for estimating diversification and sampling are more advanced than are our models for describing morphological evolution on which phylogenetic and divergence time inferences rely; thus, flexible birth-deathsampling models that can generate prior probabilities for phylogenies using temporal variation in diversification and sampling could improve the accuracy of estimated phylogenies and divergence times for analyses of fossil taxa.

\section{The Basic Problem and a General Model}

The problem that we need to address is: What is the probability that two sampled species could have a posited divergence time without one or the other having had a more recent divergence time with another sampled taxon? Every sampled species represents the tip of a lineage extending back to the origin of the clade being studied. Barring exceptional circumstances such as species-flocking (e.g., Yacobucci 1999), the lineages leading from the base of the clade to most species are united as single lineages that diverged at some point in the clade's history. Thus, the branch duration connecting any sampled Species $\mathrm{X}$ to its divergence time from the rest of the clade usually will span less time than the extended lineage leading to the base of the clade. (I use "branch duration" rather than "branch length," because the latter term also is used for expected character change along a phylogenetic branch; e.g., Lewis 2001.) Similarly, the branch duration separating the node linking Species $\mathrm{X}$ to other sampled species usually will span less time than the remainder of that extended lineage leading to the base of the clade.

Consider two hypothetical taxa, Species A and Species I (Fig. 1). Species A appears 304
Ma and Species I appears 306 Ma. The particular phylogeny we are evaluating posits that the two species diverged from a common ancestor (here represented by Species D) at $310 \mathrm{Ma}$. The maximum branch durations preceding either species are $6 \mathrm{Myr}$ for Species A and $4 \mathrm{Myr}$ for Species I (i.e., the dark gray [dark blue online] bars in Fig. 1A). One evaluation of this divergence time estimate is the probability of zero finds given the branch durations (Wagner 1995a, 2000b; Huelsenbeck and Rannala 1997). This assesses the probability that we failed to sample an immediate predecessor of Species A and Species I. However, Foote et al. (1999) make the important point that unless sampling levels are very high, most divergence times are set by sampling sister taxa rather than predecessors. Thus, if we sample any of the light gray (yellow online) lineages, then we will have a more recent divergence time and shorter branch duration for either Species A or Species I. For example, if we eventually sample Species F and correctly reconstruct the phylogeny, then we will reconstruct a divergence between Species A and Species F at 307 Ma. The branch duration leading from Species A to the rest of the clade reduces to $3 \mathrm{Myr}$; the remaining 3 Myr linking Clade $\mathrm{A}+\mathrm{F}$ to the base of the clade now is the branch duration preceding the clade including Clade $\mathrm{A}+\mathrm{F}$. Thus, estimating the probability of a branch duration and divergence time requires estimating the probability of no sampled predecessors and the probability of no additional divergences leading to sampled sister taxa.

As Bapst (2013) emphasizes, the probability of any divergence time (and its implicit branch duration and stratigraphic gap within a phylogeny) reflects three rates: origination, extinction, and sampling rates. Origination and extinction both have two effects on this probability. Origination rates provide the expected number of cladogenetic events along any branch leading to the base of the tree. Both origination and extinction affect the expected number of progeny that any one cladogenetic event will ultimately generate: as origination increases and/or extinction decreases (i.e., as net diversification increases), we expect more taxa to evolve that could become sampled sister taxa. That in turn reduces the probability of 

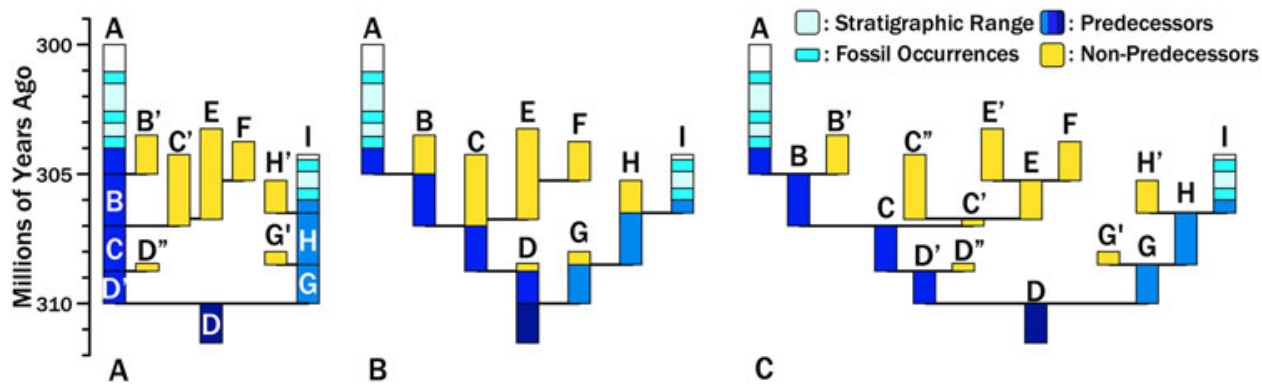

FIGURE 1. Hypothetical phylogeny for sampled lineages (black/light blue), unsampled predecessors (dark gray/dark blue), and unsampled sister taxa (light gray/yellow). A, Two sampled species diverged from unsampled Species D at $310 \mathrm{Ma}$, generating branch durations (and chronostratigraphic gaps) of $6 \mathrm{Myr}$ for Species A and 4 Myr for Species I. Increased sampling will provide earlier divergence times and shorter branch durations/chronostratigraphic gaps in one of two ways. We might sample predecessors of A or I (dark gray/dark blue). The probability of doing so is the probability of sampling an individual lineage at any one point in time. Alternatively, we might sample sister taxa of A or I (light gray/yellow). The probability of doing so reflects origination (affecting the number of possible sister taxa and the richness of sister clades), extinction (affecting the richness of sister clades and the probability of sampling individual lineages), and sampling (affecting the probability of sampling individual lineages). B, The phylogeny re-rendered as budding speciation. Each letter represents a distinct morphospecies that we would recognize before phylogenetic analysis. Note that the predecessors of A and I now are only part of the durations of ancestors such a Species B and Species H. However, the sum of predecessors (dark gray/dark blue) and the sum of possible "sisters" (light gray/yellow) remains unchanged. C, The phylogeny re-rendered as bifurcation speciation, with ancestral morphospecies being at least slightly altered at divergence so that Species B and Species B' have at least one character distinguishing them. Now predecessors and ancestors are nearly synonymous, save for the earliest unsampled members of Species A and I. However, the sum of predecessors and the sum of possible sisters remains unchanged.

deep divergences by increasing the probability of finding at least one descendant of a divergence, even if sampling rates themselves do not increase. Decreasing extinction has a similar effect, as increasing the durations of individual species increases the probability that at least one descendant of a divergence is found, even if neither origination nor sampling increase (Foote 1996). Figure 1 illustrates how diversification affects expected branch durations and divergence times. There are three branching events leading to Species A compared with only two branching events leading to Species I; thus, all else being equal, it is more probable that subsequent sampling will add a sister taxon to Species A rather than to Species I. Moreover, one of Species A's possible sisters, the $\mathrm{C}+\mathrm{E}+\mathrm{F}$ clade, offers many more opportunities for sampling than do any of the other divergences. All else being equal, the probability of sampling some descendant of that " $C$ " divergence is greater than the probability of sampling descendants of any other divergence.

These expectations are independent of particular speciation models. Re-rendering Fig. 1A as either a budding (Fig. 1B) or bifurcating (Fig. 1C) speciation model leaves the same branch durations leading from Species A and Species I to the base of the tree, and the same sum of durations for additional potential sister taxa. The difference is that "predecessors" and "ancestors" are basically identical given bifurcation, because ancestral morphotypes anagenetically change at cladogenesis, whereas predecessors include only those members of an ancestral species extant before cladogenesis under the budding model. (Under both models, the earliest members of Species A and Species I also are "predecessors.") Under the latter model, the probability of sampling an ancestor is greater than the probability of sampling a predecessor (Foote 1996). To avoid implying that ancestors necessarily become pseudoextinct at speciation, I will use the term "predecessor" for the analogues of the dark gray (dark blue online) bars in Fig. 1 throughout this paper.

Bapst (2013) and Didier et al. (2017) extend the work of Foote et al. (1999) by deriving the probability of sampling a species or any successors (i.e., a clade of unknown size) given time-homogeneous origination, extinction, and sampling. These birth-death-sampling models ( $=$ fossilized-birth-death sampling sensu Heath et al. 2014; Gavryushkina et al. 
2016; Zhang et al. 2016; Stadler et al. 2018) offer great potential as tools for assessing the branch durations and divergence times implicit to rival phylogenetic hypotheses, and thus for assessing different models of anatomical evolution favoring those rival phylogenetic hypotheses. Moreover, because we have abundant paleobiological methods for estimating origination, extinction, and sampling from the fossil record, this represents a relatively rare case in which we can empirically justify the rate parameters that we need to estimate prior probabilities of branch durations and divergence times in Bayesian analyses.

The same paleobiological methods that provide us with diversification and sampling rates also indicate that all three rates all vary over time (e.g., Connolly and Miller 2001; Foote 2001, 2003; Liow and Finarelli 2014; Alroy 2014; Finarelli and Liow 2016). What we require to accommodate interval-to-interval variation in origination, extinction, and sampling is a general model that will estimate: (1) the probability of a species appearing in some interval generating any sampled specimens either of itself or some descendant species given the diversification and sampling rates of that interval and when in that interval the first species originated; (2) the probabilities that the first species sends $1 \ldots \infty$ successors (with the successors possibly including the first species itself) into the next interval given the origination and extinction rates of that first interval; and (3) the probability that any of those successors generate at least one sampled species given the origination, extinction, and sampling rates of subsequent intervals.

\section{The Probability of Sampling a Species or Any Successors from Any One Interval}

In Figure 1, we posit that Species A and Species I are each other's closest sampled relative. The divergence time demands a branch duration and stratigraphic gap over which we failed to sample any specimens and over which no other sampled relatives diverged. If diversification and sampling rates are constant, then the expected number of sampled species or clades that should be more closely related to Species A than is Species I is $\Phi \lambda d$, where $\lambda$ is the origination rate, $\Phi$ is the proportion of branching events for which any successors are sampled (i.e., the probability of sampling a clade of unknown size sensu Bapst [2013]), and $d$ is the branch duration leading to Species A (Table 1). $\Phi$ reflects sampling $(\psi)$ and the summed species durations $(S)$ expected given $\lambda$ and $\mu$ (Foote et al. 1999). For example, the $\mathrm{C}^{\prime}+\mathrm{E}+\mathrm{F}$ clade (Fig. 1A) has $S=7.75$ lineage million years (LMyr). Bapst (2013: eq. 2) extends this logic to estimate the probability of sampling a species or any successors (i.e., a clade of unknown size) given constant $\lambda, \mu$, and $\psi$ as:

$$
\Phi=1-\sum_{K=1}^{\infty} \frac{\lambda^{(K-1)} \mu^{K}\left(\begin{array}{c}
2 K-2 \\
K-1
\end{array}\right)}{K(\lambda+\mu+\psi)^{(2 K-1)}}
$$

where $K=1 \ldots \infty$ represents the possible richness generated by any one divergence. Didier et al (2017: appendix 5; see also King in Bapst 2016) estimate $\Phi$ using a quadratic:

$$
\Phi=1-\frac{(\lambda+\mu+\psi)-\sqrt{(\lambda+\mu+\psi)^{2}-4 \lambda \mu}}{2 \lambda}
$$

Bapst's and Didier et al.'s equations generate very similar estimates of $\Phi$, and in both cases the probability of sampling a species or any successors increases as:

\section{1. $\lambda$ increases (increasing $S$ by generating more species);}

2. $\mu$ decreases (increasing $S$ by generating longer-lived species);

3. $\psi$ increases (increasing the probability of sampling per LMyr).

To estimate $\Phi$ given shifting diversification and/or sampling, we first need to estimate $\Phi^{\prime}$, the probability of sampling a clade of unknown size over some interval $i$ of duration $T_{\mathrm{i}}$ with its rates of $\lambda_{\mathrm{i}}, \mu_{\mathrm{i}}$, and $\psi_{\mathrm{i}}$. (Note that time now refers to time elapsed after a divergence, not time before a first appearance or the splitting from a common predecessor.) First, we calculate the probability that one lineage will result in $K=0 \ldots \infty$ lineages at any time from $t=0 \ldots T$ (Fig. 2). When $\lambda \neq \mu$ :

$$
P[K=0 \mid \lambda, \mu, t]=\frac{\mu \times\left(e^{(\lambda-\mu) t}-1\right)}{\left(\lambda \times e^{(\lambda-\mu) t}\right)-\mu}
$$


TABLE 1. Glossary of terms and variables of importance in this work.

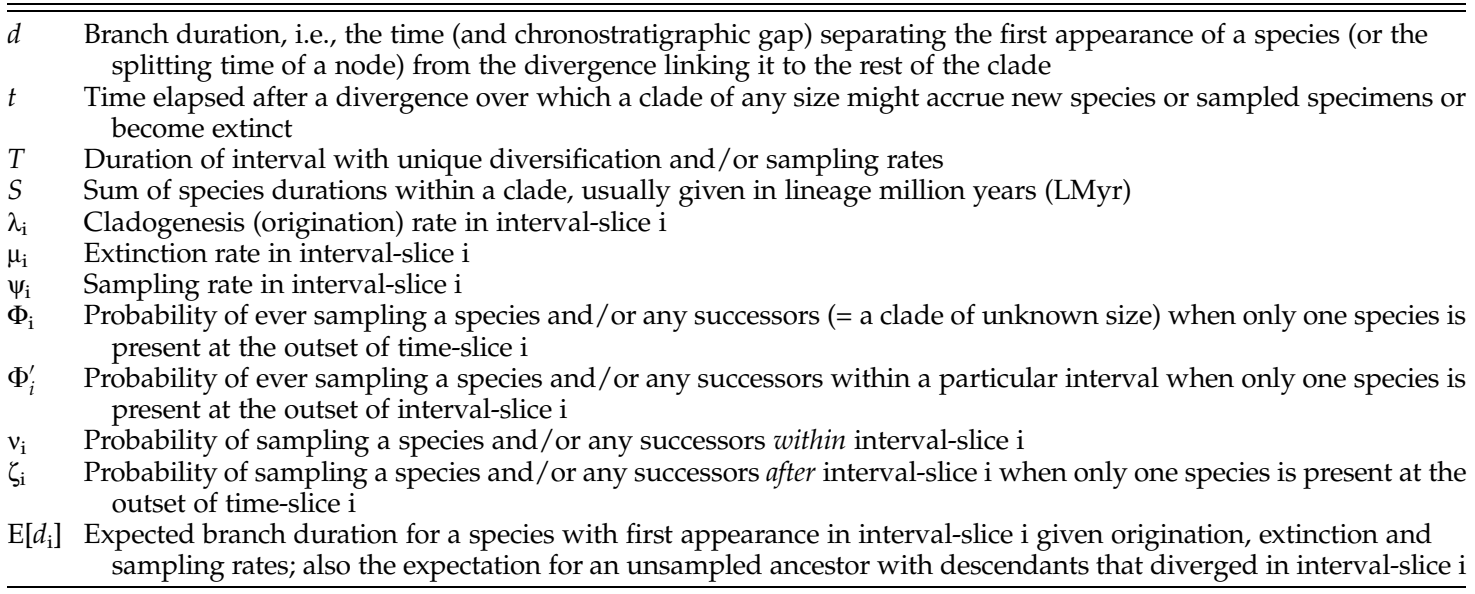

(Raup 1985: eq. A13) and

$$
\begin{aligned}
P[K \geq 1 \mid \lambda, \mu, t] & =\left(1-\frac{\mu \times\left(e^{(\lambda-\mu) t}-1\right)}{\left(\lambda \times e^{(\lambda-\mu) t}\right)-\mu}\right) \\
& \times\left(1-\frac{\lambda \times \mu \times\left(e^{(\lambda-\mu) t}-1\right)}{\mu \times\left(\left[\lambda \times e^{(\lambda-\mu) t}\right]-\mu\right)}\right) \\
& \times\left(\frac{\lambda \times \mu \times\left(e^{(\lambda-\mu) t}-1\right)}{\mu \times\left(\left[\lambda \times e^{(\lambda-\mu) t}\right]-\mu\right)}\right)^{K-1}
\end{aligned}
$$

(Raup 1985: eq. A13, A17). When $\lambda=\mu$ :

$$
P[K=0 \mid \lambda, \mu, t]=\frac{\lambda t}{1+\lambda t}
$$

(Raup 1985: eq. A12), and

$$
P[K \geq 1 \mid \lambda, \mu, t]=\frac{(\lambda t)^{K-1}}{(1+\lambda t)^{K+1}}
$$

(Raup 1985: eq. A14). Figure 2 illustrates these in nine combinations of rates over some interval with $T=2.5$. Both $\lambda$ and $\mu$ give expectations over $t=1$ (e.g., rates per million years for an interval of $2.5 \mathrm{Myr}$ ).

Integrating over the individual probability curves for $K$ successors of one species gives the $E\left[t_{K}\right]$, the expected amount of time within interval $i$ in which $K$ successors of the original species are present (Fig. 3). We can use this to estimate $E[S]$, the expected sum of durations within an interval:

$$
E[S]=\sum_{K=1}^{\infty}\left(K \times E\left[t_{K}\right]\right)
$$

that is, the number of lineages $(K)$ times the expected time with that number of lineages $\left(E\left[t_{N}\right]\right.$ ) summed over all possible $K$. (Because $E\left[t_{K}\right]$ rapidly declines to very low numbers, the summation can be quickly truncated.) If we have Interval A with $\lambda=\mu=0.4 \mathrm{Myr}^{-1}$ (Fig. 3A), then:

$$
\begin{gathered}
\mathrm{E}\left[S_{A}\right]=(1 \times 1.250)+(2 \times 0.625)+(3 \times 0.313) \\
+(3 \times 0.156)+(4 \times 0.078) \ldots \\
=2.5 \text { LMyr }
\end{gathered}
$$

If we have Interval B with $\lambda=0.566$ and $\mu=$ 0.4 (Fig. 3B), then:

$$
\begin{aligned}
\mathrm{E}\left[S_{B}\right]= & (1 \times 1.125)+(2 \times 0.716)+(3 \times 0.456) \\
& +(3 \times 0.290)+(4 \times 0.185) \ldots \\
= & 3.1 \mathrm{LMyr}
\end{aligned}
$$

A first approximation of the probability of sampling a lineage present at the outset of any interval $X$ or any successors to that species is:

$$
\Phi_{X}^{\prime}=1-e^{-\psi_{X} E\left[S_{X}\right]}
$$

Therefore, if $\psi_{\mathrm{A}}<1.24 \times \psi_{\mathrm{B}}$, then we expect to sample more clades diverging at the onset of Interval B than we would for Interval A. 

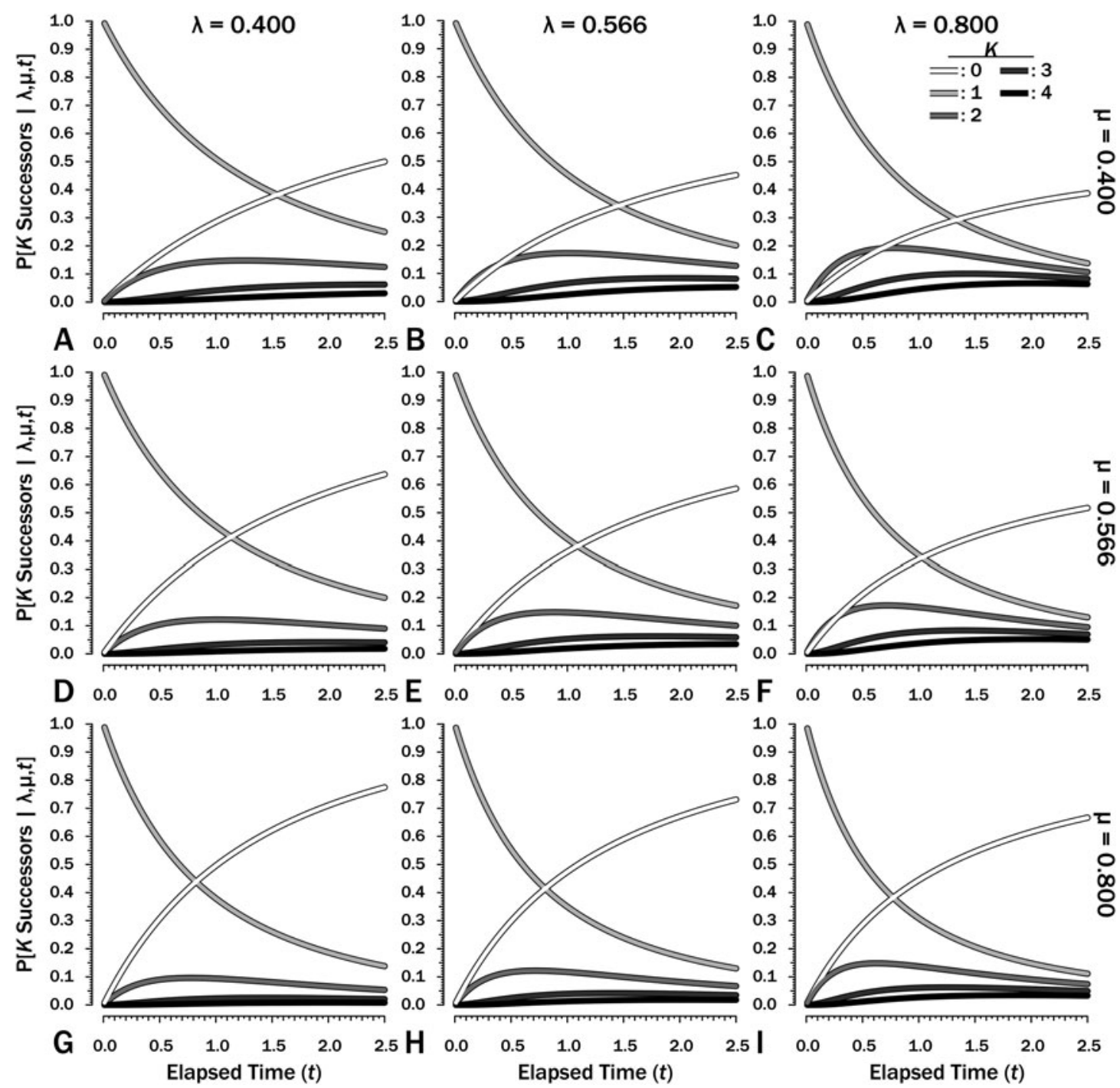

FIGURE 2. The probability of there being $K=0 \ldots 4$ successors of a species extant at time $t=0$ over an interval with duration $T=2.5$ given different combinations of origination $(\lambda)$ and extinction $(\mu)$ rates. Note that rates increase by a factor of 1.414 $\left(2^{0.5}\right)$ between adjacent frames. Note that the successors might include the original species at any point in time.

Simulations show that eq. (7) overestimates $\Phi^{\prime}$. At $\lambda=\mu=\psi=0.4$ and $T=2.5$, eqs. (6 and 7) estimate $\Phi^{\prime}=0.632$, whereas simulations starting using these same parameters generate sampled species in only $53.3 \%$ of runs (see also Supplementary Fig. S1). The culprit is the failure of eq. (6) to account for total extinction within the interval eliminating any possibility of additional sampling regardless of the remaining expected $S$ (see Supplementary Fig. S2). One solution is to divide intervals into $m$ interval-slices so that each per-slice $\psi$ is low and then estimate: (1) $v_{i}$, the probability of a species extant at the outset having sampled members or successor in interval-slice $i$ (eq. 7); and (2) $\zeta_{i}$, the probability of a lineage present at the outset of interval-slice $i$ having sampled successors in interval-slices $i+1$ to $m$. The former parameter, $v$, is the probability of finding a species and/or any successors in an interval-slice:

$$
v_{i}=1-e^{-\psi_{i} E\left[S_{i}\right]_{i}}
$$

where $\psi_{\mathrm{i}}$ is the sampling rate for the interval, $S_{\mathrm{i}}$ is the expected total progeny in the interval-slice, 

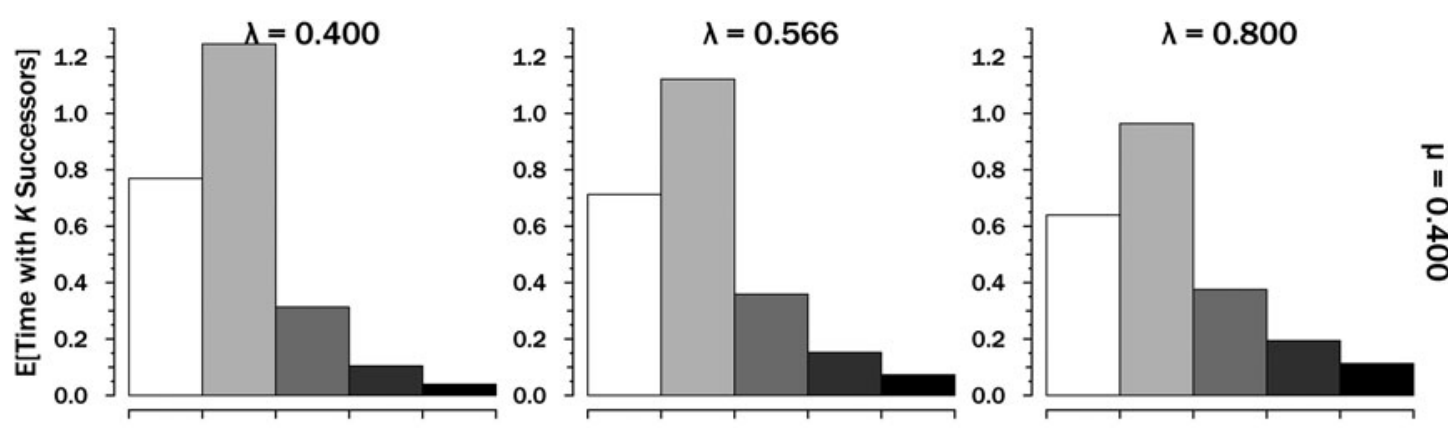

A
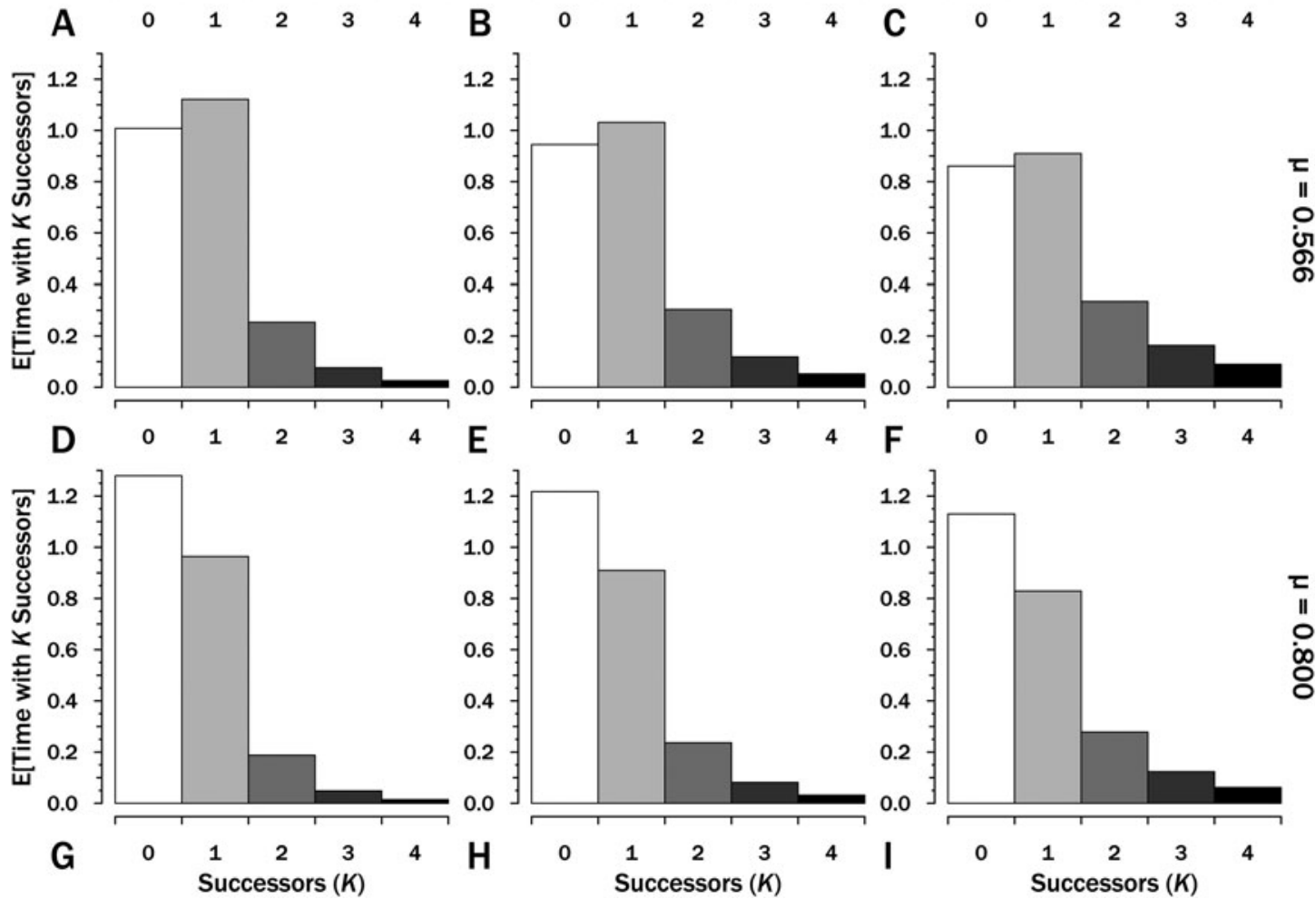

FIGURE 3. The expected time with $K=0 \ldots 4$ successors (including the original species) given interval duration $T=2.5$. These are the areas under each curve for the corresponding $K$, origination $(\lambda)$ and extinction $(\mu)$ in the corresponding panels in Fig. 2. The sum of expected lineage durations, $S$, is the sum of $K \times \mathrm{E}\left[T_{K}\right]$. Thus at $\lambda=0.4$ and $\mu=0.4(\mathrm{~A}), \mathrm{E}[S]=(1 \times 1.25)+$ $(2 \times 0.313)+(3 \times 0.104)+(4 \times 0.039) \ldots=2.5$ LMyr.

and $t_{\mathrm{i}}$ is duration of the interval-slice. The latter parameter, $\zeta$, is the probability of $K=1 \ldots \infty$ total progeny yielding at least one sampled species times given "future" $\lambda, \mu$, and $\psi$ multiplied by the probability of the initial species having $K=1 \ldots \infty$ successors (again, possibly including the original species) at the end of the intervalslice $i$ given $\lambda_{\mathrm{i}}$ and $\mu_{\mathrm{i}}$ (Fig. 4):

$$
\begin{aligned}
\zeta_{i}= & \sum_{K=1}^{\infty}\left(P\left[\geq 1 \text { Finds } \mid K, \Phi_{i+1}^{\prime}\right]\right. \\
& \left.\times P\left[K \text { Successors } \mid \lambda_{i .}, \mu_{i}\right]\right)
\end{aligned}
$$

For the final interval-slice $m, \zeta_{m}=0$, because there are no opportunities to sample successors later in the interval. Thus, $\Phi_{m}^{\prime}=v_{\mathrm{m}}$, and we can solve $\zeta_{1 \ldots(m-1)}$ recursively. The maximum possible value for $\zeta_{i}$ is the probability of any successors surviving interval-slice $i$ : at $\lambda=\mu=0.4$ and $T_{\mathrm{i}}=0.25$, the probability of extinction is 0.091 (Fig. 5A) and the maximum possible $\zeta_{i}$ is 0.909. Now:

$$
\Phi_{i}^{\prime} \cong 1-\left(\left[1-v_{i}\right] \times\left[1-\zeta_{i}\right]\right)
$$



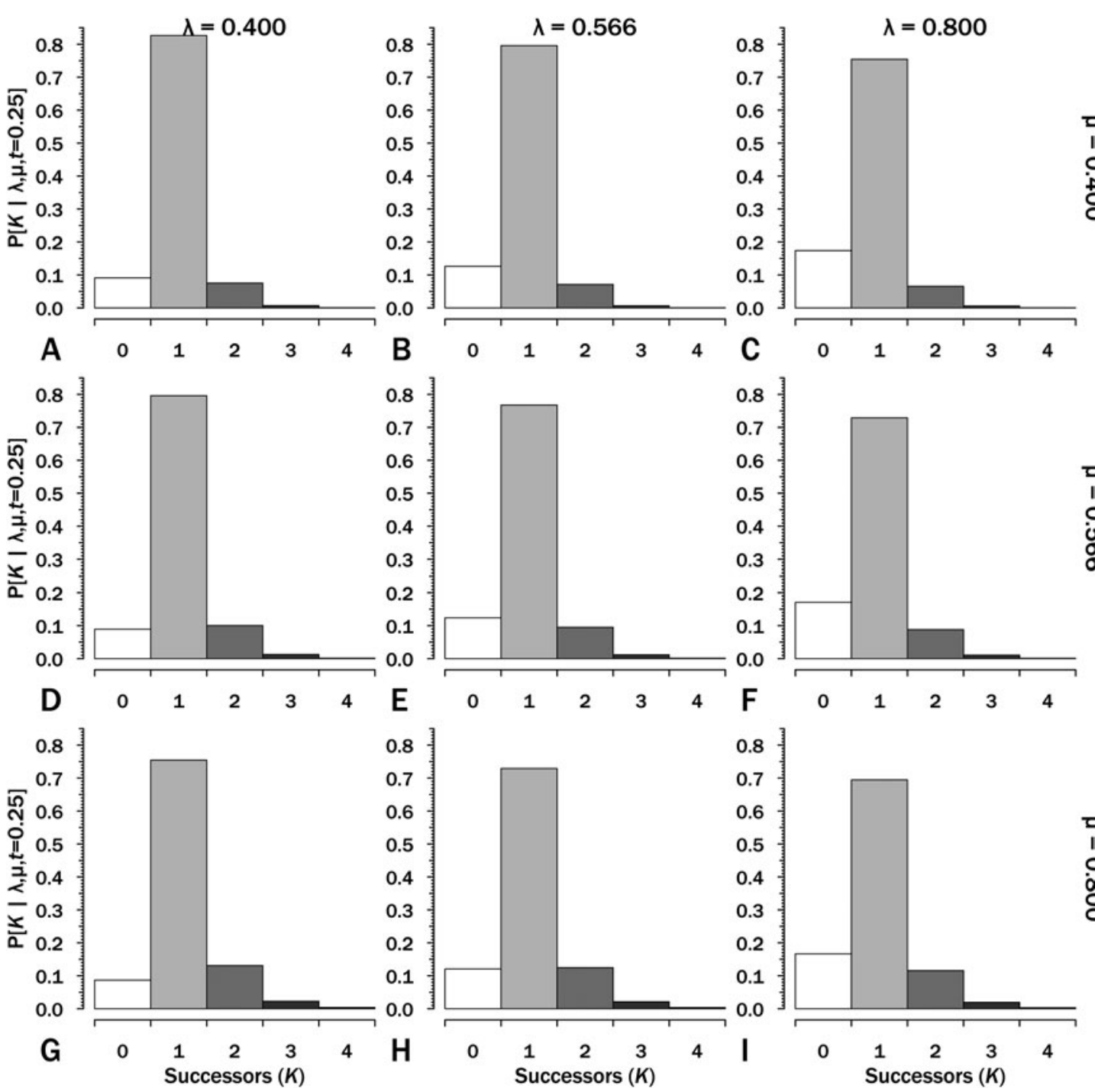

FIGURE 4. The probability of a species extant at the outset of an interval with particular origination $(\lambda)$ and extinction $(\mu)$ having $K=0 \ldots 4$ successors extant $0.25 \mathrm{Myr}$ later. The probability of the species or any successor generating a sampled species in the subsequent $0.25 \mathrm{Myr}$ is conditioned on the probability of $K=0 \ldots \infty$ survivors going into the next interval-slice.

This explicitly accommodates the probability that a lineage present at the outset of an interval is extinct with no successors by (say) the $5^{\text {th }}$ of $m=10$ interval-slices.

Simulations (Fig. 5, Supplementary Fig. S1) show that eq. (9) rapidly converges to appropriate estimates of $\Phi_{1}^{\prime}$ at $m \geq 10$ over the range of diversification parameters used in Figures 2-5. Thus, if we typically have intervals of (say) $2.5 \mathrm{Myr}$ over which we have estimates of $\lambda, \mu$, and $\psi$, then $100 \mathrm{kyr}$ interval-slices should be adequate to estimate $\Phi_{1}^{\prime}$.
Another useful aspect of calculating $\Phi_{i}^{\prime}$ for multiple slices within an interval is that divergences could happen at any time within an interval if origination is continuous through time. Suppose that the precision of divergence dates that we will consider are in $100 \mathrm{kyr}$ increments: that is, we will ask whether a divergence happened at $450.1 \mathrm{Ma}, 450.0 \mathrm{Ma}, 449.9 \mathrm{Ma}$, etc. If these divergences are within a $2.5 \mathrm{Myr}$ chronostratigraphic interval, then we then we need to know $\Phi_{1 \ldots 25}^{\prime}$. $\Phi_{i}^{\prime}$ will decrease as i increases: we have both less time to accrue 

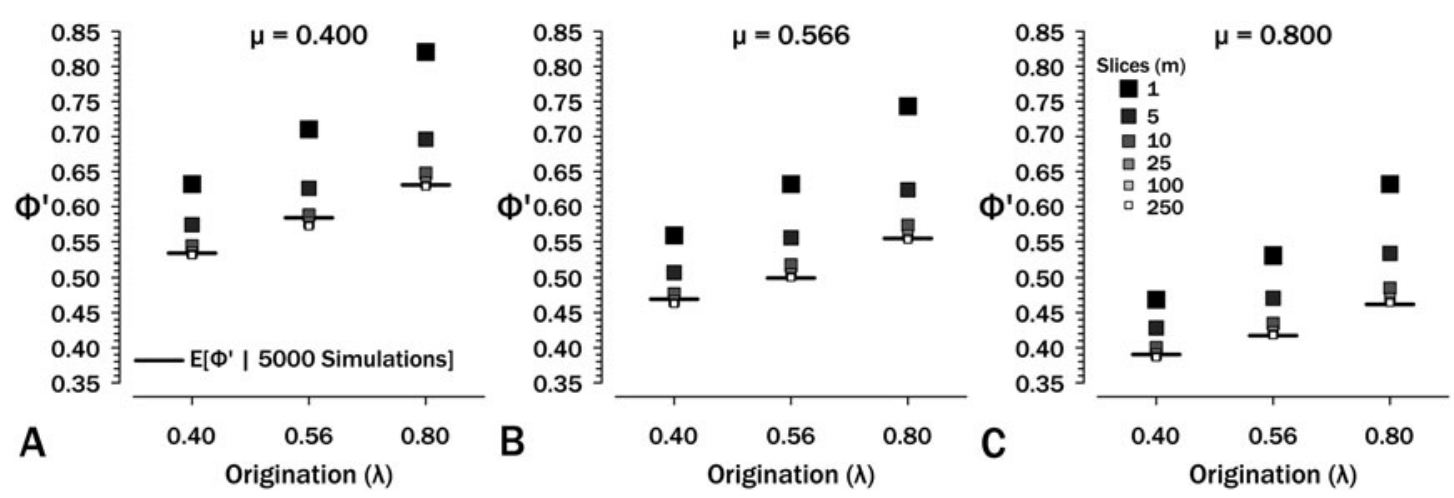

FIGURE 5. The effect of interval-slice length on overestimate of $\Phi^{\prime}$. Horizontal bars denote expected $\Phi^{\prime}$ given 5000 simulations at the appropriate origination $(\lambda)$ and extinction $(\mu)$ rates. Intervals durations are set to $T=2.5$, so at $m=250$ slices, each $t_{\mathrm{i}}=0.001$. See also Supplementary Fig. S3.

lineage durations and less time in which to sample them. If the interval has $\lambda=\mu=\psi=$ $0.4 \mathrm{Myr}^{-1}$, then: $\Phi_{1}^{\prime}=0.536, \Phi_{13}^{\prime}=0.383$, and $\Phi_{25}^{\prime}=0.039$. Thus, even if the probability of a divergence is the same at any point within the interval, the probability of a divergence that generates a sampled species within that interval declines over time.

\section{The Probability of Sampling a Species or Successors over 2+ Intervals with Different Diversification and/or Sampling Rates}

The exercise above applies sampling within one interval. However, this is just the particular case where all $m$ diversification and sampling rates are the same and $v_{1}=v_{2} \ldots=v_{m}$. We can calculate eq. (9) just as easily using $v_{1} \neq v_{2} \ldots \neq v_{m}$, as will be the case if either of the diversification rates or the sampling rate varied in every interval-slice. Similarly, $\zeta_{i}$ is just as easy to calculate if $\lambda_{i}$ and/or $\mu_{i}$ differ in every intervalslice. What I will do here is intermediate: interval-slices from different intervals will use particular values of $\lambda, \mu$, and $\psi$ so that each $v_{\mathrm{i}}$ within an interval is identical and each $\zeta_{i}$ within an interval uses the same values of $\lambda$ and $\mu$. The one difference is that $\zeta_{m}$ will be greater than zero for all intervals save the last, and all $\zeta_{i}$ from those intervals will reflect subsequent shifts in diversification and/or sampling.

Suppose that we are analyzing a clade of Ordovician gastropod species known through the Katian. Suppose further that data from the Paleobiology Database coupled with an external stratigraphic database allow us to estimate separate diversification and sampling rates for gastropods as a whole for three divisions of the Katian, corresponding to Bergström et al.'s (2009) Ka1, Ka2 + Ka3 (hereafter: $\mathrm{Ka} 2-3)$, and Ka4: $\lambda_{\mathrm{Ka} 1}=0.269, \lambda_{\mathrm{Ka} 2-3}=0.348$, $\lambda_{\mathrm{Ka} 4}=0.312 ; \mu_{\mathrm{Ka} 1}=0.293, \mu_{\mathrm{Ka} 2-3}=0.495, \mu_{\mathrm{Ka} 4}=$ $0.514 ;$ and, $\psi_{\mathrm{Ka} 1}=0.147, \psi_{\mathrm{Ka} 2-3}=0.155, \psi_{\mathrm{Ka} 4}=$ 0.122 (Fig. 6). These parameters come from capture-mark-recapture analysis (Connolly and Miller 2001; Liow and Nichols 2010) using Paleobiology Database data, with the analysis modified to allow for log-normal distributions of sampling rates (Wagner and Marcot 2013) and each $\psi_{\mathrm{Ka}}$ the median of those log-normal distributions. Each 100 kyr interval-slice within any one interval will have the same probability of a divergence in that interval-slice being sampled immediately: $v_{\mathrm{i} \cdot \mathrm{Ka} 1}=1.46 \times 10^{-2}, v_{\mathrm{i} \cdot \mathrm{Ka} 2-3}=$ $1.53 \times 10^{-2}, v_{i \cdot K a 4}=1.20 \times 10^{-2}$ (Fig. 6A). Each $100 \mathrm{kyr}$ interval-slice within an interval also shares the same probability of a species present at the outset having $0 \ldots \infty$ successors at the outset of the next 100 kyr interval-slice (Supplementary Fig. S3). However, $\zeta_{i}$ for a Ka2-3 divergence (i.e., the probability of sampling the species or successors in subsequent intervalslices) now reflects diversification and sampling in both Ka2-3 and Ka4 (Fig. 6). This in turn means that we now have $\Phi_{i}$ for each interval-slice within all three intervals: that is, the probability of ever sampling a descendant of a divergence occurring in that $100 \mathrm{kyr}$ interval-slice at some point in the time range encompassed by our study. Simulations using 

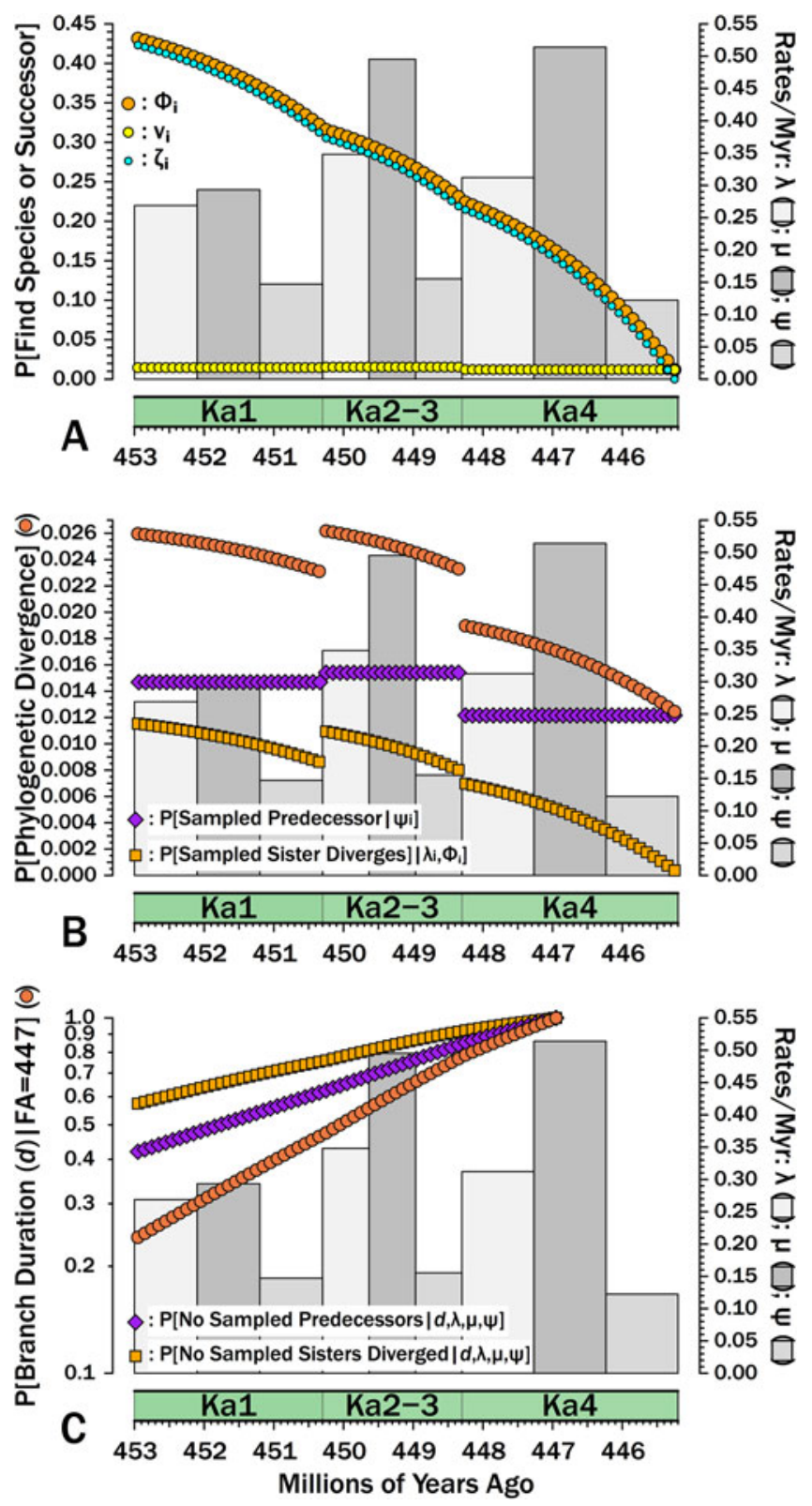

FIGURE 6. Breaking down estimates of branch duration probabilities given empirically estimated rates of origination $(\lambda)$, extinction $(\mu)$, and sampling $(\psi)$. Gray bars give rates for each interval ( $2^{\text {nd }} y$-axis). A, The components of $\Phi_{i}$, the probability of sampling a species appearing in interval-slice $i$ or any successors of that species (i.e., a clade of unknown size). $v_{i}$ (yellow dots) gives the probability of sampling that species or any successors in interval-slice $i$. $\zeta_{\mathrm{i}}$ (light blue dots) gives the probability of sampling any successors of that species (including the original species) in interval-slice $i+1$ or any time afterward before the end of the Katian. $\Phi_{i}$ reflects the probability of doing either and therefore is $\Phi_{i}=1-\left(\left[1-v_{i}\right] \times\left[1-\zeta_{i}\right]\right)$. B, The exact probability of a branch duration leading to a sampled species or to a node linking sampled species starting in interval-slice i. The probability of sampling a predecessor (purple) is the probability of sampling the one predecessor of a species or node extant in interval-slice $i$. The probability of sampling a sister taxon (dark orange) is the probability of a divergence giving rise to a sister taxon with $1+$ sampled species in interval-slice i. The probability of one or the other happening (sienna) is the probability of a phylogenetic divergence in interval-slice i given that we have some sampled species with a first occurrence after interval-slice $\mathrm{i}$ (or a node linking sampled species that diverges after interval-slice i). C, The probabilities of branch durations (sienna) preceding Salpingostoma richmondensis, which is first known from early in the Aphelognathus divergens conodont Zone. This is broken down into the probability of duration $d$ with no divergences to sampled sister taxa (orange) and the probability of no sampled precursors (purple). The expected branch duration (where $\mathrm{P}[d]=0.50)$ is $3.2 \mathrm{Myr}$. 
the same origination, extinction, and sampling rates per interval indicate that eqs. (7a) and (8) combined generate accurate estimates of $\Phi_{i}$ for all interval-slices (Supplementary Fig. S4). This in turn indicates that eq. (6) is accurately estimating $E[S]$ (expected summed durations evolving from a divergence) for different interval-slices given heterogeneous $\lambda$ and $\mu$.

\section{The Probability of Branch Durations and Divergence Times Given Shifting Rates of Diversification and/or Sampling}

We now can estimate the probability that a branch duration leading to a sampled species (or to the basal divergence of a sampled clade) diverges from the rest of the clade in any particular interval-slice. Following the gastropod example above, suppose that we are analyzing a group of bellerophontoids through the Katian. One of the species is Salpingostoma richmondensis, which is first known from rocks at the base of the Aphelognathus divergens conodont Zone in Ka4. What is the probability that $S$. richmondensis diverged from its closest relative before the Katian? Based on Gradstein et al. (2012), we assign an earliest appearance date of $447 \mathrm{Ma}$ for $S$. richmondensis, that is, about halfway through Ka4. (I will disregard uncertainty in the time-scale here.) Given that the Katian starts at $453 \mathrm{Ma}$, we are positing a minimum branch duration of $d=6 \mathrm{Myr}$. The probability of failing to sample a predecessor of $S$. richmondensis over $d=6 \mathrm{Myr}$ is the probability of missing it in every intervalslice $i$ :

$$
e^{-\sum_{i=1}^{F A-1} \psi_{i} d_{i}}
$$

where FA-1 is the interval-slice preceding the first appearance (here, the interval-slice corresponding to $447.1 \mathrm{Ma}$ ), $d_{1}$ is the time encompassed by each interval-slice (here, $100 \mathrm{kyr}$ ), and $\psi_{i}$ is the sampling rate of that interval. (Fig. 6B gives the complement of this, i.e., the probability of sampling a predecessor per interval-slice). Given the sampling rates from above, this comes to $p=0.420$.

The probability of there being no divergences leading to a sister taxon of S. richmondensis sampled in the Katian reflects the three separate origination rates and $\Phi_{i}$ at any given point in time:

$$
\prod_{i=1}^{F A-1} \sum_{n=1}^{\infty}\left(P\left[n \text { branchings } \mid \lambda_{i} d_{i}\right] \times\left[1-\Phi_{i}\right]^{n}\right)
$$

where $\mathrm{P}\left[n\right.$ branchings $\left.\mid \lambda_{\mathrm{i}}\right]$ is the Poisson probability of $n$ cladogenetic events in an intervalslice given the origination rate of that interval and the duration of each interval-slice (here, $100 \mathrm{kyr})$, and $\left[1-\Phi_{i}\right]^{n}$ is the probability of $n$ divergences yielding no sampled sister taxa. Here, this generates $p=0.574$. We usually will get almost identical results estimating the probability of no divergences leading to sampled sister taxa as:

$$
e^{-\sum_{i=1}^{F A-1} \Phi_{i} \lambda_{i} d_{i}}
$$

because the probability of just one branching event is nearly equal to the probability of there being any branching events. However, at origination rates comparable to those estimated for major radiations, the probability of two branching events over even short intervals is great enough that not allowing for $n=2$ yields notable underestimates of the probability of a sampled sister taxon. (Note that even with very high $\lambda$, we can truncate the summation by $n=5$.)

The complement of eq. (11) is the probability that any given interval-slice includes a divergence leading to a sampled sister taxon of $S$. richmondensis (Fig. 6B). This increases not just as $\Phi$ increases (Fig. 6A), but also as $\lambda$ increases: thus, the probability of a divergence leading to a sampled sister taxon of $S$. richmondensis peaks early in $\mathrm{Ka} 2-3$, despite the edge effect of ending the study after the next interval (Fig. 6B). Because $\psi_{\mathrm{Ka} 2-3}$ is only marginally higher than $\psi_{\mathrm{Ka} 1}$ and $\psi_{\mathrm{Ka} 4}$ is less than $\psi_{\mathrm{Ka} 1}$, this necessarily is driven by the $\lambda_{\mathrm{Ka} 2-3}$ being greater than $\lambda_{\mathrm{Ka} 1}$. The combination of elevated $\lambda$ and elevated $\psi$ in Ka2-3 also means that these interval-slices have the highest probabilities of providing S. richmondensis's divergence from either a sampled predecessor or a sampled sister taxon.

We now calculate the probability of branch duration $d$ as the probability of no sampled predecessors (i.e., one minus eq. 10) times the 
probability of no divergences leading to sampled sister taxa (i.e., one minus eq. 11). Thus, the probability that $S$. richmondensis has a divergence time from other bellerophontoids no later than the base of the Katian given the shifting diversification and sampling rates of the Katian (and without the possibility of sampling post-Katian sister taxa) is $0.420 \times 0.574=$ 0.241 (Fig. 6C). Finally, note that if we do not include any post-Katian species, then the expected branch duration for $S$. richmondensis $(\mathrm{E}[d])$, that is, the point where the probability of the branch duration is 0.5 , is $3.1 \mathrm{Myr}$.

\section{Effects of Simple Shifts in Sampling and/or Diversification Rates on Expected Stratigraphic Gaps and Branch Durations}

The specific effects of each parameter are difficult to appreciate when origination, extinction, and sampling all vary from one interval to the next, as in the Katian gastropod example. Moreover, the short span of time encompassed by the Katian data creates a major edge effect: $\Phi_{i}$ steadily declines throughout the Katian because no post-Katian descendants of Katian divergences can be sampled. Most studies include many more than one stage and thus might have many intervals without such an edge effect. Indeed, just how long before the conclusion of a study (or before the total extinction of a clade) we need to go before this edge effect disappears is in itself a question of interest.

Therefore, I will present simple examples in which (with one exception) two of the rate parameters are held constant while one varies over time. This will emphasize how the particular parameters affect the probabilities of branch durations and divergence times. I will first cover shifts in sampling with constant diversification. I then will cover some simplified versions of major evolutionary events given constant sampling. The latter examples are particularly important, not simply because they emphasize the strong effects of both origination and extinction rates on expected branch durations and stratigraphic gaps within a phylogeny, but because paleobiologists often target species spanning such events when conducting phylogenetic analyses. In each example, I will use 6 intervals of 2.5 Myr each, with estimates of $\Phi$ based on $100 \mathrm{kyr}$ intervalslices. The "standard" diversification rates are set to $0.4 \mathrm{Myr}^{-1}$ so that we expect one event per interval. In all cases, I will use different median sampling levels that range from an expectation of one sample per interval to one sample per 100 intervals. To avoid the edge effect from the Katian gastropod example, all of the examples (save one) are followed by 12 intervals with "normal" $\lambda, \mu$, and $\psi$.

For each combination of parameters and parameter shifts, I present the following: (1) $\Phi$, the probability of sampling a species or any successors (= a clade of unknown size); (2) the probability of a divergence leading to a sampled sister taxon given expectation $\lambda_{i} \Phi_{i} ;$ (3) the probability of truncating a branch duration in interval-slice i either by sampling a predecessor or from the divergence of a subsequently sampled sister taxon; and (4) the expected branch duration $(E[d])$ given a sampled species with that first appears in interval-slice $i$. The last represents the branch duration where the probability of encountering either a sampled predecessor or the divergence time of a sampled sister taxon reaches 0.5 . Note that this $\mathrm{E}[d]$ also applies to the expected branch duration leading to a node that diverges in interval-slice $i$.

Finally, for $\Phi$ over time, I also include the results from 10,000 simulations (dots in Figs. $7 \mathrm{~A}, 8)$. Each simulation begins with a single lineage, with both lifetimes and descendants per time-slice allowing for shifts in either diversification or sampling. Lifetimes and progeny of descendants are simulated the same way, albeit using rates specific to their lifetimes. Intervalspecific rates of sampling are used when sampling changes. The results show the proportion of simulated lineages that generate $1+$ sampled species.

\section{Effects of Shifts in Sampling Rates $(\psi)$}

Although there are several evolutionary scenarios positing general shifts in origination and/or extinction rates, temporal patterns in sampling rates $(\psi)$ do not have analogous simple models. The biggest control on $\psi$ is the amount of available sedimentary rock from the right environmental type (Raup 1972; Sheehan 1977; Peters and Foote 2001; Smith 2001a), 

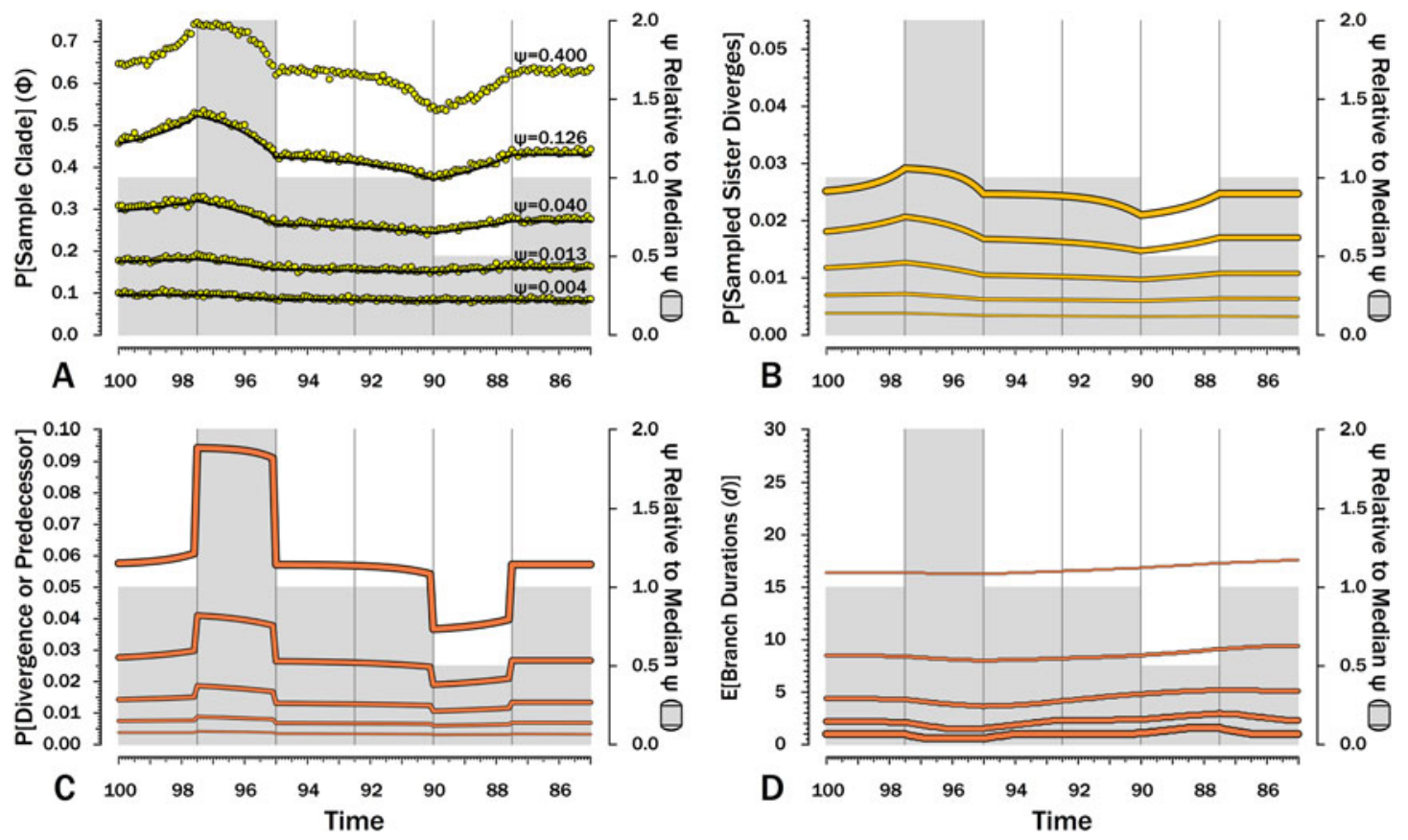

FIGURE 7. Effects of variable sampling over time with constant origination and extinction $(\lambda=\mu=0.40)$. Five median sampling rates $(\psi)$ are illustrated, with $\psi$ relative to the median given on by the gray bars ( $2^{\text {nd }} y$-axis $)$. The six intervals of $2.5 \mathrm{Myr}$ are preceded by six others with median $\psi$. A, $\Phi$, the probability of sampling a species or any successors given that the species is extant in an interval-slice. Yellow dots give expected $\Phi$ given 10,000 simulations using $\psi($ and $\lambda$ and $\mu$ ) of the intervals of a species lifetime. B, The probability of a sampled sister species diverging in each interval-slice. C, The probability of a phylogenetic divergence in each interval-slice from either sampling a predecessor or from the divergence of a subsequently sampled sister taxon. D, Expected durations $(d)$ for a species first appearing in each interval-slice. This also applies to a node that diverged in the same interval-slice. $\mathrm{E}[d]$ is the branch duration where $\mathrm{P}[$ no ancestors or divergences] reaches 0.50 (see Fig. 6C).

which can vary fairly haphazardly over time. In the absence of any such templates, I use two shifts in $\psi$, where one interval has twice the median sampling rates and another has half the median sampling rates (Fig. 7). A key feature is that the effects of shifts in sampling on the probability of sampling a species or any descendants $(\Phi)$ precedes shifts in $\psi$ (Fig. 7A): compared with species appearing in other intervals, species appearing shortly before elevated sampling have a higher probability of surviving to and/or having descendants in the interval of high sampling even if they do not have elevated probabilities of numerous descendants and/or long durations. Conversely, $\Phi$ is depressed before the fourth interval, because species arising then have much lower probability of having descendants present during "normal" sampling intervals. We see the same patterns in reverse within the intervals of elevated and depressed $\psi: \Phi$ declines over the second interval, because species arising late in that interval have less time to generate descendants during the time of elevated sampling than do species arising early in the interval. Similarly, $\Phi$ increases over the fifth interval, because species arising late in the interval have a higher probability of having successors present during intervals of normal $\psi$. These patterns are mirrored in the actual probabilities of a sampled sister taxon arising in each interval-slice simply because $\lambda$ is constant here (Fig. 7B). Finally, it is the absolute shifts in $\psi$ that seem to be important: simply doubling low $\psi$ has relatively little impact because $2 \psi$ never is high enough in such cases to greatly improve the probability of a clade member being sampled before the clade becomes extinct.

The exact probability that an earlier-sampled species or clade diverges from other sampled members of the clade in any interval-slice 

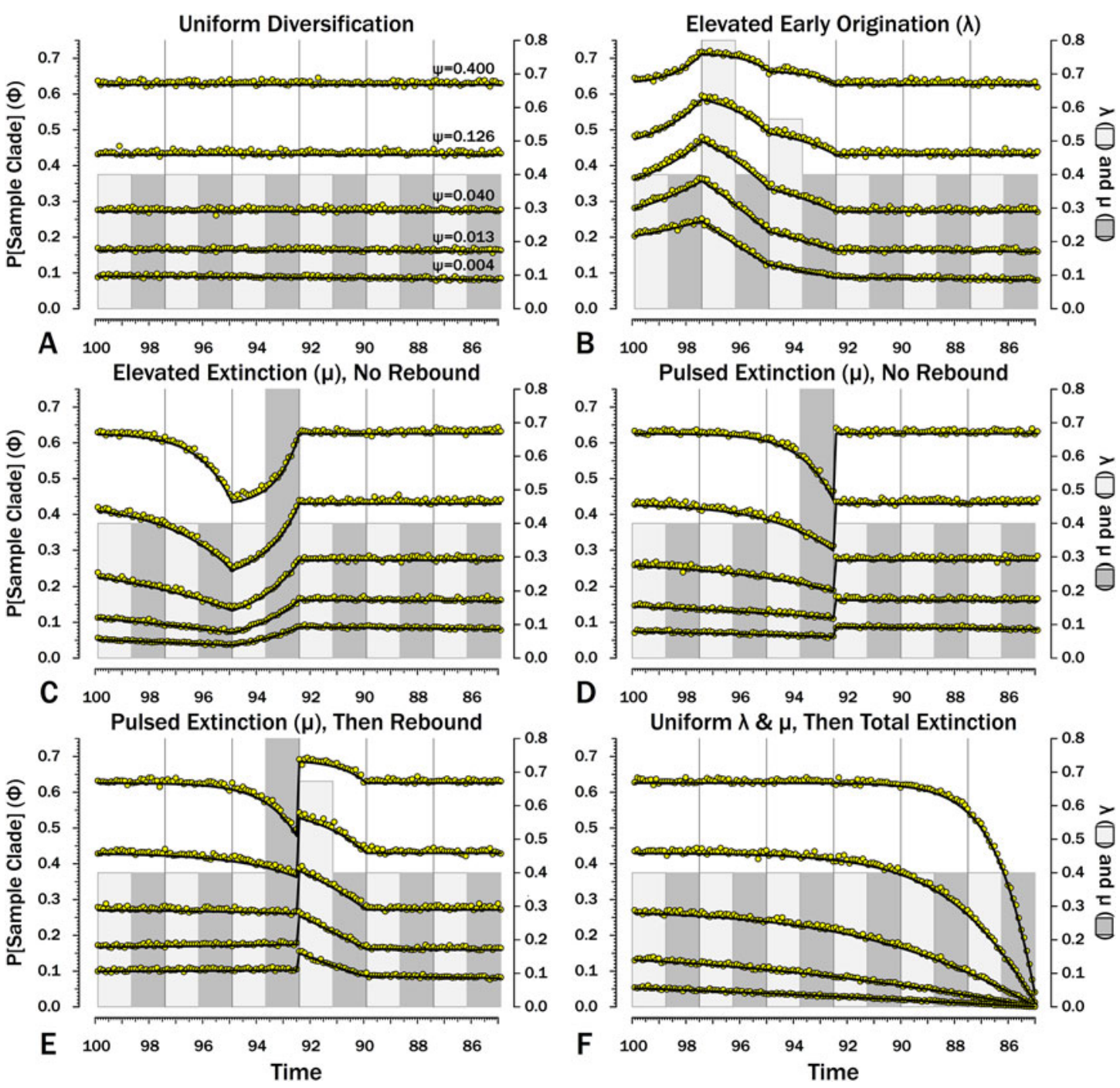

FIGURE 8. Probabilities of sampling clades of unknown size $(\Phi)$ under exemplar models of diversification. Origination, extinction, and sampling rates are per millions of years, with each interval $2.5 \mathrm{Myr}$ long. Each $\Phi_{\mathrm{i}}$ is for a $100 \mathrm{kyr}$ slice. Black lines indicate $\Phi$ based on text eq. (9) and the rates of origination ( $\lambda$, in light gray) and extinction ( $\mu$, in dark gray) of that time-slice and subsequent time-slices. For each curve, one of five sampling rates $(\psi)$ is assumed for the entire duration. For pulsed extinction, the gray bar shows the average stage rate, with $\mu_{i}=0.4$ for the first 24 slices and $\mu_{i}=10.4$ for the final interval-slice, giving average $\mu=0.8$. Yellow dots represent the results of 10,000 simulations and give the proportion of runs in which a lineage appearing at that time ultimately generates a sampled specimen given subsequent diversification.

reflects both $\psi$ at that point in time (i.e., the probability of sampling a predecessor) and subsequent $\psi$ (i.e., the probability of sampling a sister clade). The primary shifts (Fig. 7C) strongly reflect the changing probability of sampling a predecessor, particularly when $\psi$ is high and the probability of a sampled predecessor or the divergence of a sampled sister taxon is high. However, changes in $\Phi$ in response to reduced $\psi$ in later intervals cause the probability of a sampled divergence date to grade from one interval to the next, rather than simply be flat throughout an interval (see, e.g., Wagner 2000b). As above, this effect is muted when $\psi$ is low, simply because absolute values are more important than relative values here.

When $\psi$ is high, the expected branch durations $(E[d])$ increase or decrease rapidly around intervals of depressed or elevated sampling 
(Fig. 7) and then return to "normal." When $\psi$ is low, shifts in $\mathrm{E}[d]$ are extended over much longer period of time. Reduced sampling lengthens expected branch durations for taxa evolving well after the low- $\psi$ interval, whereas elevated sampling reduces expected branch durations for taxa evolving well after the low- $\psi$ interval. Indeed, at the lowest $\psi$ used here, the effects of the elevated $\psi$ interval on $\mathrm{E}[d]$ extend up to taxa appearing during (or just before) the low- $\psi$ interval! This stated, the effects are fairly muted at low- $\psi$, at least when $\psi$ varies by only factors of two. Finally, simulations indicate that our estimates of $\Phi$ accurately predict the probability of sampling at least one descendant of any given divergence when $\psi$ varies, regardless of the typical rate.

Effects of Shifts in Diversification Rates $(\lambda$ and $\mu)$

I use five shifts in diversification rates to stereotype five basic macroevolutionary scenarios:

1. a burst of high speciation with constant extinction;

2. an interval of elevated continuous extinction and constant origination;

3. an interval ending with elevated pulsed extinction and constant origination;

4. an interval ending with elevated pulsed extinction followed by a rebound interval of elevated origination; and

5. sudden total extinction ending a clade.

I also include constant rates of $\lambda$ and $\mu$ (Figs. 8A, $9 \mathrm{~A}, 10 \mathrm{~A}, 11 \mathrm{~A})$. This provides a comparison for assessing the importance of shifting rates and also allows comparison with both the Bapst (2013) and Didier et al. (2017) estimates of $\Phi$ (Table 2).

Constant Diversification and Sampling.When diversification and sampling are constant over time, the estimates of $\Phi$ that allow for temporal variation in diversification and sampling rates are nearly identical to estimates assuming constant rates (Table 2). The greatest difference is seen when $\psi$ is very low. As discussed later, this reflects the fact that the edge effect of not allowing sampling 13+ intervals later begins to affect the probability of sampling sister taxa at very low levels of sampling.
Intervals of Elevated Origination.-Here, $\lambda$ doubles in the second interval, and then decreases by 1.414 times over both of the next two intervals to return to the "normal" level. Elevating $\lambda$ raises $\Phi$ not just in the intervals of high $\lambda$, but also in preceding intervals (Fig. 8B). The latter effect becomes more pronounced as $\psi$ decreases: at $\psi=0.4$ (50\% taxon sampling given $\mu=0.4$ and $P$ [taxon sampling] $=\frac{\psi}{\mu+\psi} ;$ Foote 1996), $\Phi$ at the onset of the interval preceding diversification is close to that expected given normal rates (Fig. 8A); however, given $\psi=0.004(\sim 0.99 \%$ taxon sampling given $\mu=0.4), \Phi$ at the onset of the preceding interval is twice that expected given normal rates. Accordingly, the probability of a sampled clade diverging in the interval preceding a radiation is greater than the probability of a sampled clade diverging after that radiation, even if the origination rates themselves are the same before and after (Fig. 9B).

The effects of elevated $\lambda$ on expected branch durations and stratigraphic gaps within a tree $(\mathrm{E}[d])$ also are more dramatic when $\psi$ is low than when $\psi$ is high (Figs. 10B, 11B). When $\psi$ is high, expected sampled predecessors make long branch durations and stratigraphic gaps improbable given either high or low $\lambda$ (see, e.g., Foote 1996). On the other hand, when $\psi$ is low, divergence times usually are truncated by sampled sister taxa and the increased probability of sampled sister taxa when or before $\lambda$ is high reduces expected branch durations. Thus, the effect of elevated $\lambda$ on $\mathrm{E}[d]$ is more pronounced under low $\psi$ than under high $\psi$.

Interval of Continuous Elevated Extinction.Here, $\mu$ is twice normal for one interval. Elevated $\mu$ causes both $\Phi$ and expected sampled sister taxa to decrease in the intervals leading up to it (Figs. 7C, 8C). This reflects the decreased probability of a lineage having numerous and/or long-lived progeny. As $\psi$ decreases, the effect of elevated $\mu$ on $\Phi$ extends further back in time before the extinction interval. However, $\Phi$ increases as the extinction interval progresses, reflecting the increasing probability of extant species having surviving successors that might be sampled in subsequent intervals.

Intervals of elevated extinction increase the probability of stratigraphic gaps (Fig. 9C) and 

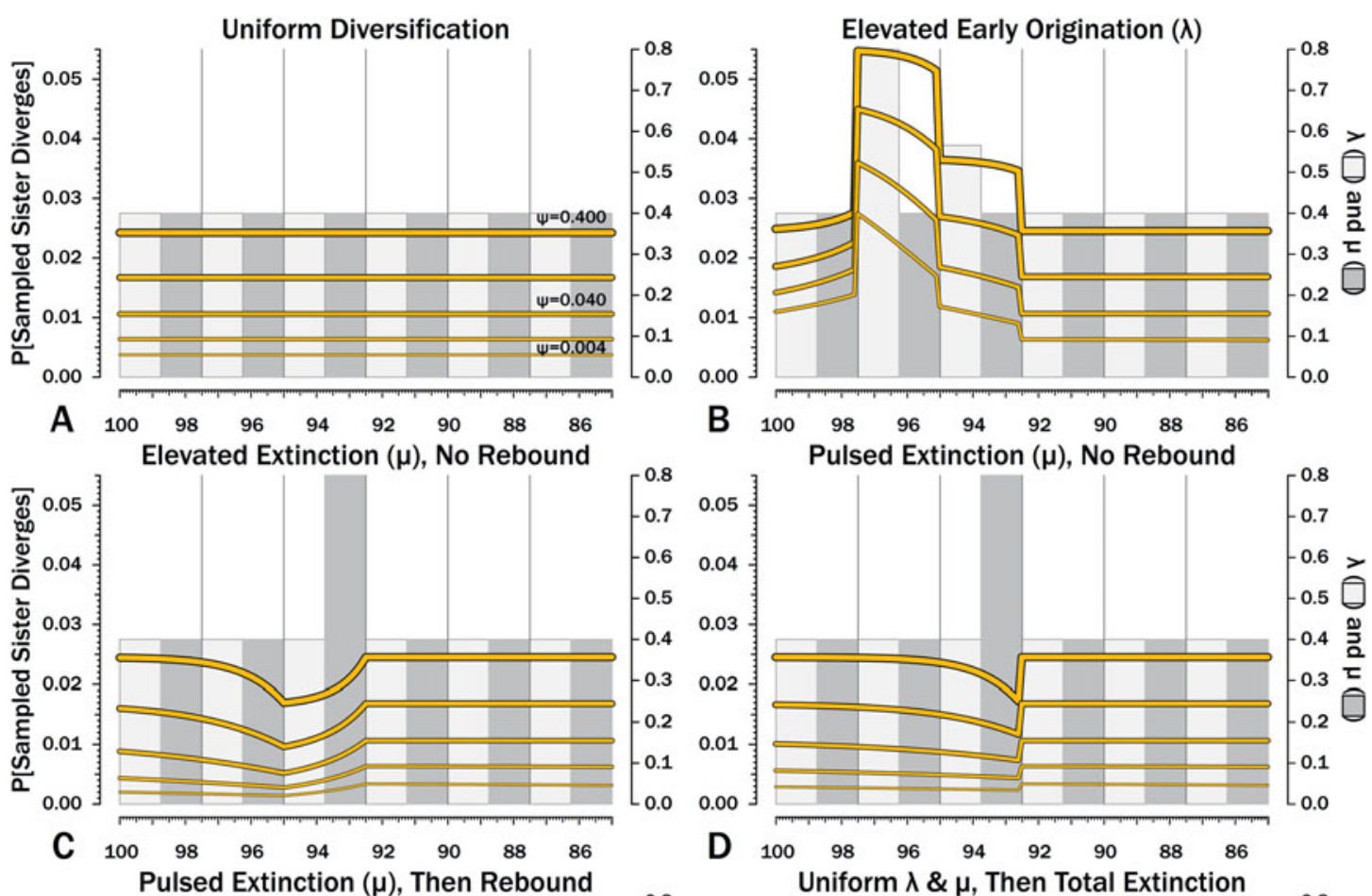

Pulsed Extinction $(\mu)$, No Rebound
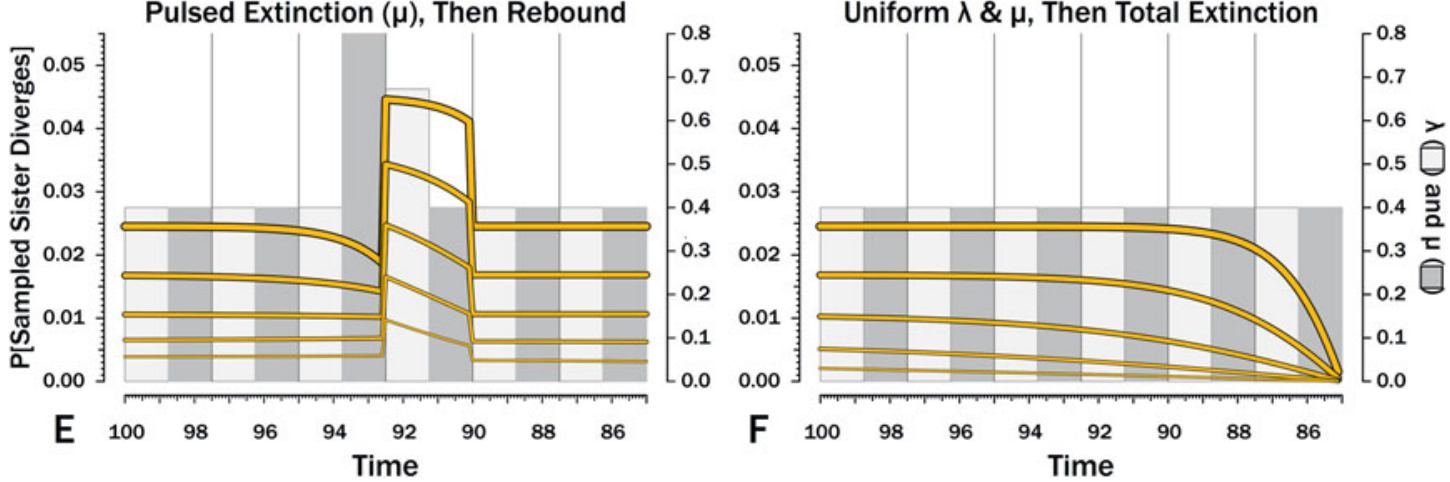

FIGURE 9. Probabilities of a divergence that ultimately generates a sampled species given the subsequent origination, extinction, and sampling rates.

thus inflate expected branch durations and stratigraphic gaps for species first appearing either before or after the interval of extinction (Figs. 10C, 11C). This reflects the increased probability of sister taxa going extinct before generating a sampled species. The effect becomes more pronounced as $\psi$ decreases and the probability of setting a divergence time with a sampled predecessor decreases: not only is $E[d]$ higher during the extinction interval, but $\mathrm{E}[d]$ is also inflated in earlier and later intervals (Fig. 8C vs. $8 \mathrm{~A}$ at the same $\psi$ ).

Interval Ending in Pulsed Elevated Extinction.-There is considerable evidence that some major extinctions occur as pulses at the end of intervals (Marshall 1995a; Jin et al. 2000; Wang and Marshall 2004; Wang et al. 2014). Therefore, I model pulsed extinction here by setting $\mu_{i}=10.4$ for the final intervalslice and $\mu_{i}=0.4$ for the first 24 interval-slices. The probability of extinction for an individual species present at the outset of the highextinction interval is the same as in the continuous elevated-extinction example (0.86), but with the bulk of the extinction concentrated in the final interval-slice. This generates a similar decline in $\Phi$ and expected sampled divergences as we see given elevated continuous extinction 

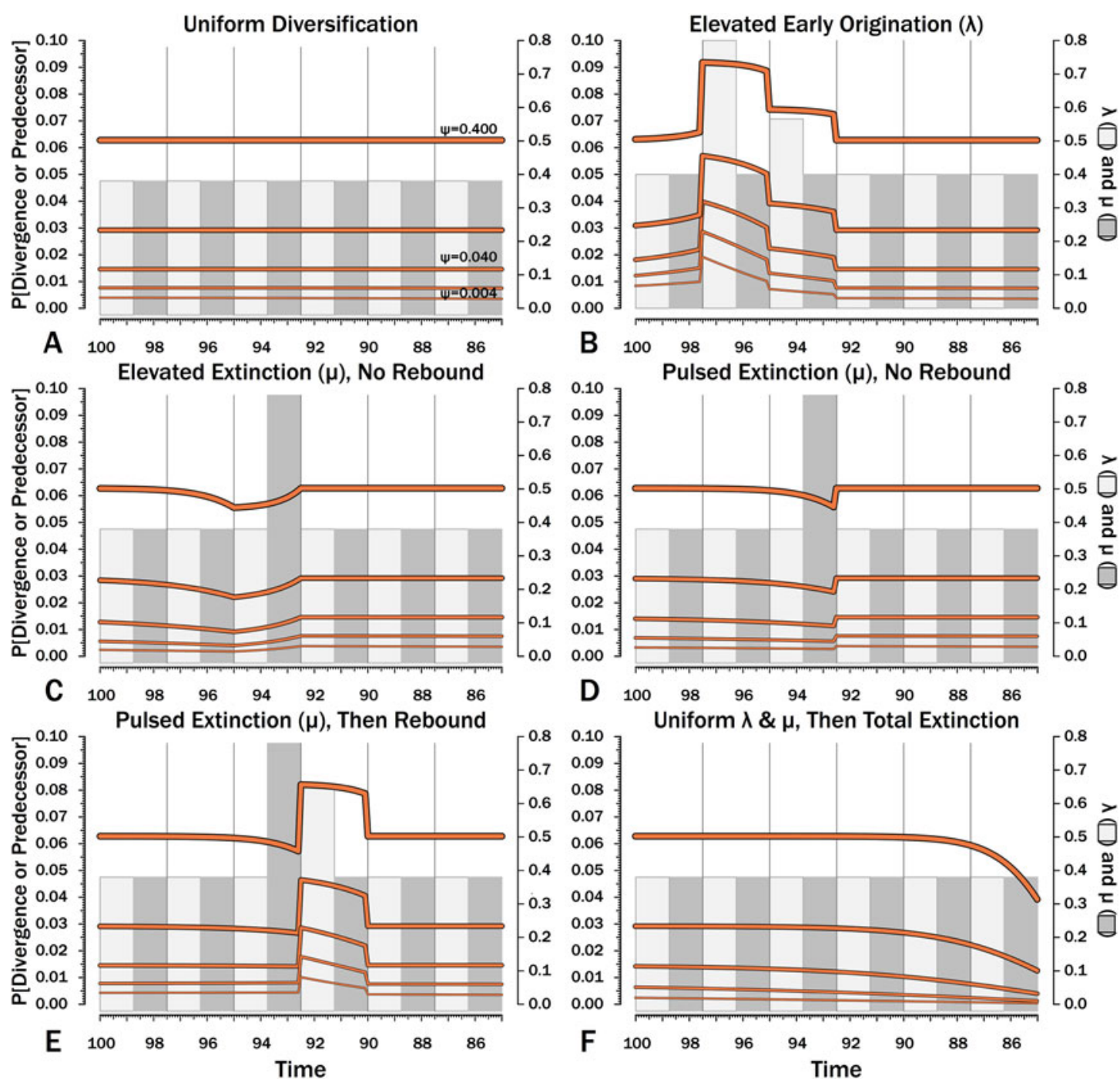

FIGURE 10. Probabilities of setting a phylogenetic divergence in a particular $100 \mathrm{kyr}$ interval-slice, either by sampling a predecessor or by the divergence of a sister taxon that is sampled in that interval-slice or in some subsequent one. The complements of these probabilities are the probabilities of a stratigraphic gap within a phylogeny over the particular interval-slices. The product of these probabilities over any set of interval-slices gives the prior probability of a branch duration spanning that set of interval-slices.

(Figs. 10D, 11D vs. 10C, 11C), but with the decline in $\Phi$ now shifted so that the minimum values are at the end of the extinction interval rather than at the outset. Correspondingly, the rebound to "normal" $\Phi$ and expected sampled divergences is immediate rather than protracted. The differences in both offset and the rebound reflect species appearing in the middle of the interval not having appreciably better probabilities of having surviving successors than species appearing or existing at the outset of the interval.
The amount of time with depressed $\Phi$ before the extinction also is lower with pulsed extinction than with continuous extinction of the same magnitude, particularly when $\psi$ is low. The effect on expected branch durations and stratigraphic gaps at any given sampling level $\psi$ is muted under pulsed extinction relative to continuous extinction (Figs. 9D vs. 9C and $10 \mathrm{D}$ vs. 10C). Not only are the maximum deviations from "normal" lower, but the departures from normal begin and end closer to the extinction event. 

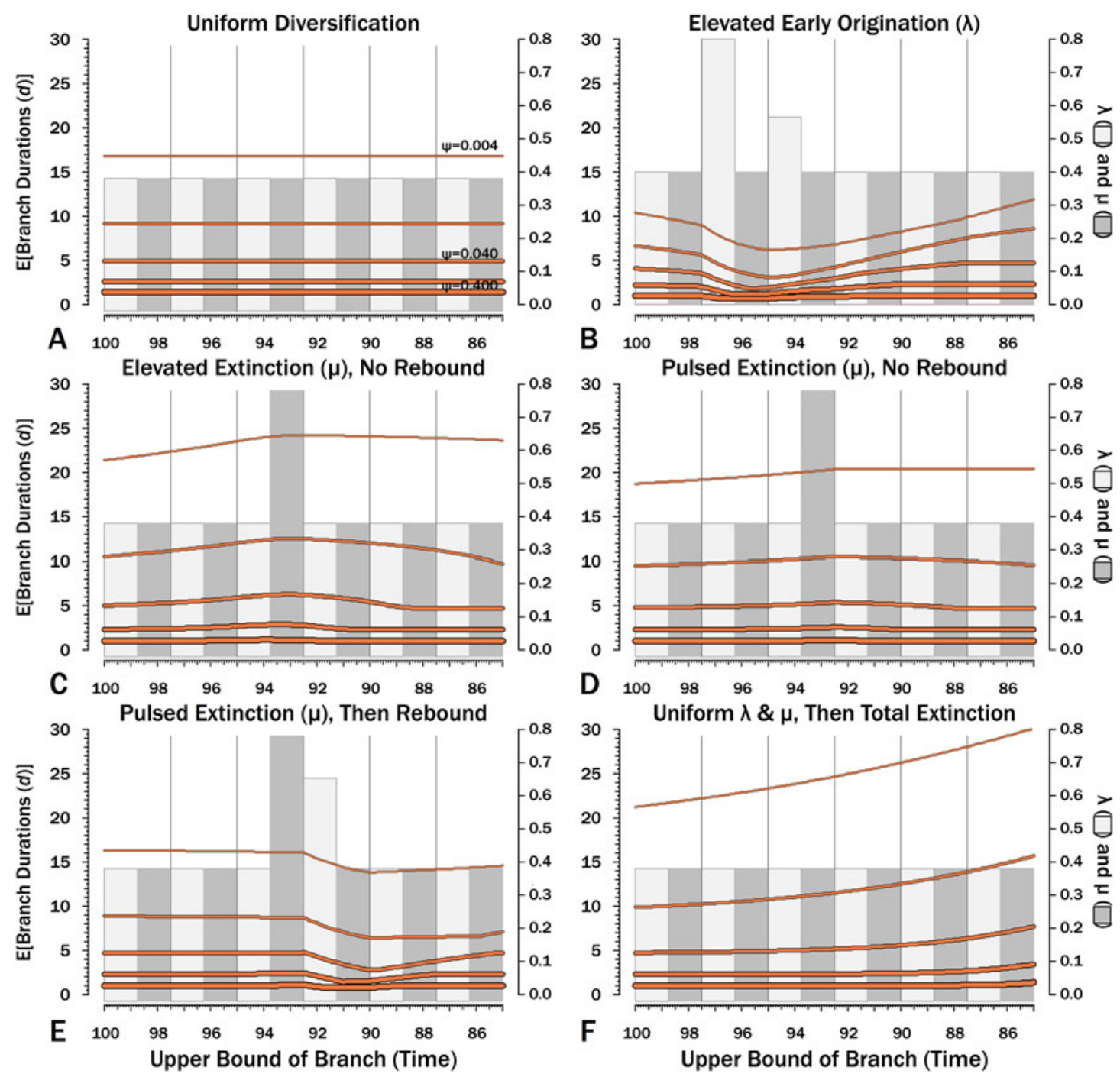

FIGURE 11. Expected branch durations (E[d]) for a species first appearing in an interval-slice or for an unsampled ancestor diverging in that interval-slice. $\mathrm{E}[d]$ is the branch duration where $\mathrm{P}[d \mid \lambda, \mu, \psi]=0.5$. Note that $\mathrm{E}[d]$ at low $\psi$ allows for several preceding intervals with "normal" diversification and sampling rates.

TABLE 2. The probability of sampling a clade of unknown size given origination and extinction $=0.4$ and different levels of sampling $(\psi)$. $\Phi_{\mathrm{B}}$ gives the global estimate for a clade given Bapst (2013); $\Phi_{\mathrm{D}}$ gives the global estimate given Didier et al (2017); and med. $\Phi_{\mathrm{i}}$ gives the median $100 \mathrm{kyr}$ estimate that allows for variation in diversification and sampling.

\begin{tabular}{lccc}
\hline \hline$\Psi$ & $\Phi_{\mathrm{B}}$ & $\Phi_{\mathrm{D}}$ & med. $\Phi_{\mathrm{i}}$ \\
\hline 0.400 & 0.6180 & 0.6180 & 0.6259 \\
0.126 & 0.4260 & 0.4260 & 0.4297 \\
0.040 & 0.2702 & 0.2701 & 0.2716 \\
0.013 & 0.1628 & 0.1627 & 0.1618 \\
0.004 & 0.0955 & 0.0951 & 0.0867 \\
\hline
\end{tabular}

Pulsed Elevated Extinction followed by Rebound in Origination.-Intervals following elevated extinction often show rebounds with elevated origination (e.g., Miller and Sepkoski 1988; Lu et al. 2006; Foote et al. 2018). The probability of sister taxa evolving in the last interval and then surviving the extinction are the same as in the prior example. However, there now is a much higher probability that any species evolving before the extinction that do have surviving successors will generate a sampled descendant, which dampens the drop in $\Phi$ (Figs. 8E, 9E vs. 
$8 \mathrm{D}, 9 \mathrm{D})$. This results in a mixing of the expected phylogenetic patterns preceding extinctions and radiations: the extinction reduces the probability of sampled sister taxa diverging before the extinction and subsequent, which offsets the preradiation decrease in expected branch durations (Figs. 10E, 11E vs. 10B, 11E); however, the elevated probability of sampling the successors of any survivors dampens the expected increase in branch durations induced by the extinction (Figs. 10E, 11E vs. 10D, 11D). As in other scenarios, low $\psi$ results in more time elapsing after the rebound before we return to "normal" branch durations and stratigraphic gaps. In particular, $\mathrm{E}[d]$ decreases going back in time (Fig. 11E) until the rebound interval, and then rapidly increases. Going further back in time, $\mathrm{E}[d]$ increases slightly rather than decreases (as expected when there is no rebound), with the time of increase greater at low $\psi$ than at high $\psi$.

Complete Extinction (or End of Study Edge Effect).-Whole clades might go extinct during pulsed extinctions, which ends the chance to sample subsequent successors. For example, in a phylogenetic analysis of nonavian dinosaurs or ammonites from the Cretaceous, there is no opportunity for an unsampled Maastrichtian species to leave Paleocene or Eocene species that would document that particular divergence. Therefore, divergences that are not sampled in the Maastrichtian are never sampled. This has strong effects on branch durations. Even if $\lambda, \mu$, and $\psi$ are static before the extinction event, $\Phi$ declines markedly before the extinction (Fig. 8F). Because divergences generating sampled sister taxa become rarer (Fig. 9F), expected branch durations increase as taxon first appearances approach the extinction (Figs. 10F, 11F).

The effect of $\psi$ on $\Phi$ and expected sampled sister taxa seen for elevated extinction rates above becomes even more pronounced given total extinction. Under high $\psi, \Phi$ begins to decline notably only about 1.5 intervals $(=1.5$ times the average species duration) before the extinction. However, at the lowest $\psi, \Phi$ is only half of "normal" (Fig. 8F vs. 8A) six intervals (again, six times the average species duration) before the extinction. We see a corresponding effect on expected branch durations (Fig. 11F).
When $\psi$ is high, $\mathrm{E}[d]$ is low until just before the extinction: even though we have little chance of sampling late divergences in any way, we have a high probability of sampling the predecessors of those late-appearing species that we do sample. When $\psi$ is low and we do not expect to sample many direct predecessors, $\mathrm{E}[d]$ just before the extinction can be nearly twice $\mathrm{E}[d]$ under the same "normal" diversification and sampling rates (Fig. 11E vs. 11A), simply because of our inability to sample survivors of the extinction.

The sudden extinction scenario here is the same as edge effect that excluding post-Katian species induces on the expected branch durations for an analysis of pre-Hirnantian gastropods only. As also noted earlier, this edge effect can be seen in Table 2. At the two lowest sampling levels, median $\Phi$ given homogeneous $\lambda, \mu$, and $\psi$ is lower when we allow for temporal variation than when we do not. At the secondlowest level (where we expect to sample about $3 \%$ of taxa), $\Phi_{i}$ drops below the "global" estimate over 18 intervals before the end of the calculations. At the lowest $\psi, \Phi_{i}$ is less than the estimates assuming constant diversification and sampling at least 30 intervals before the conclusion.

\section{Discussion}

Simulations illustrated in Figures 7 and 8 and Supplementary Figure S4 indicate that the approach here does well at predicting the probability of sampling a species and/or any of its descendants given "future" sampling and diversification. This reflects eq. (6) and its building blocks adequately converting $\lambda_{i}$ and $\mu_{i}$ to $S_{i}$, the expected summed durations of a lineage or clade members within an interval-slice. As this is the most complicated portion of estimating probabilities of unsampled branch durations, this gives us some confidence that the approach will work with real data using even more complex interval-to-interval variation in diversification and sampling. Therefore, I will discuss first some implications for tree-based studies, and then outline some further modifications that we can make to accommodate evolutionary scenarios not considered earlier. 
Shifts in Net-Diversification Rates and Shifts in Sampling Rates Have Similar Effects on the Prior Probability of Sampled Sister Taxa

Comparing Figure 7 to Figures 8-11 corroborates a conclusion that necessarily follows from the works of Foote et al. (1999), Bapst (2013), and Didier et al. (2017): increasing netdiversification rates has the same general effect as increasing sampling rates, whereas decreasing net-diversification rates has the same general effect as decreasing sampling rates. The primary difference in how net diversification and sampling affect branch durations is that whereas both affect the probability of sampling sister taxa, only sampling rates affect the probability of sampling predecessors. A corollary to this is that the relative effects of shifts in diversification rates on expected branch durations and divergence times increase as overall sampling decreases: if sampled predecessors only infrequently terminate branch durations, then we require changes in $\lambda$ and/or $\mu$ to increase or decrease the probability of sampling a sister taxon. Conversely, if we had $100 \%$ taxon sampling, then sister taxa would never set divergence times, as we would find all predecessors.

\section{Phylogenetic Signor-Lipps Effect}

Elevated extinction rates over short periods of time coupled with imperfect sampling create "gradual" series of last appearances in the fossil record (i.e., the Signor-Lipps effect; Signor and Lipps 1982; Raup 1986). This extends to phylogenetic patterns: because elevated extinction decreases the probability that any descendant of a divergence will ever be sampled, we expect there to be fewer nodes on phylogenies leading up to major extinctions even if rates of $\lambda, \mu$, and $\psi$ are static up to that extinction. The magnitude of the effect increases both with the severity of the extinction and with the paucity of the fossil record. The latter is particularly important. Consider ammonites and nonavian dinosaurs, both of which are entirely eliminated by the $\mathrm{K} / \mathrm{Pg}$ extinction. Because ammonites almost certainly have much higher $\psi$ than do dinosaurs (Foote and Sepkoski 1999; Bapst et al. 2016), we expect the K/Pg to induce less "distortion" in the branch durations and stratigraphic gaps within Cretaceous ammonite phylogeny than within Cretaceous dinosaur phylogeny (Figs. $8 \mathrm{~F}, 9 \mathrm{~F}, 10 \mathrm{~F}, 11 \mathrm{~F})$.

Dinosaurs offer a possible warning case here. Sakamoto et al. (2016) note a decreasing frequency of nodes in nonavian dinosaur phylogeny through the latter half of the Cretaceous. They attribute this to prolonged decline in $\lambda$. However, we expect at least part of this to be due to the $\mathrm{K} / \mathrm{Pg}$ extinction eliminating nonavian dinosaurs and creating a phylogenetic Signor-Lipps effect that reduces Late Cretaceous $\Phi$ and thus expected nodes among Late Cretaceous taxa. The question then becomes: How far back in time might this effect have stretched? Answering this requires first answering another question: What are typical sampling rates for dinosaur? Starrfelt and Liow (2016) use the TRiPS method to estimate the overall sampling probability of dinosaur species to be greater than 0.5. As per-taxon sampling probability equals $\frac{\psi}{\mu+\psi}$ (Foote 1996), this implies that sampling rates exceed extinction rates and that any Signor-Lipps effect on phylogeny will be restricted to the latest Maastrichtian (Figs. 8F-11F). However, Bapst et al. (2016; see also Benson et al. 2018) use Foote's (1997) method to estimate that dinosaur $\psi$ is about 60 times lower than dinosaur $\mu$. This is most comparable to the expectations given the lowest $\psi$ used here. Bapst et al. estimated $\mu=0.935$, which in turn implies an average dinosaur species duration of 1.1 Myr. At the lowest sampling levels examined earlier, the phylogenetic Signor-Lipps effect extends back 30 times the average species lifetime. For dinosaurs, that would mean reduced sampling of sister taxa extending back over $30 \mathrm{Myr}$, that is, to the Campanian. Relative sampling could be even lower than that for dinosaurs. Distributions of $\psi$ among contemporaneous mammal species follow log-normal distributions (Wagner and Marcot 2013; see also Foote 2016), and the sampling rates estimated by methods such Foote's 1997 one typically are 10 to 100 times higher than median (= geometric mean) log-normal rates. If so, then the Signor-Lipps effect on $\Phi$ caused by the $\mathrm{K} / \mathrm{Pg}$ extinction could be more severe than any case illustrated here. On the other hand, interest in extinction patterns leading up to the $\mathrm{K} / \mathrm{Pg}$ might create 
unusually high $\psi$ in the Maastrichtian, which in turn should increase the proportions of nodes found in the Late Cretaceous (see, e.g., Starrfelt and Liow 2016: Fig. 1). This would be similar to the "pull of the recent" (Raup 1979) effect, in which the high sampling of extant taxa increases the representation of recent divergences on phylogenies (Heath et al. 2014). Thus, assessing the effects of the total extinction of nonavian dinosaurs requires not just the typical sampling rates, but also the heterogeneity in those sampling rates over time.

Low Sampling Will "Blur" Radiations Back in Time

When using appearance or occurrence data, incomplete sampling has the same effect on the apparent timing of radiations as it does on extinctions, albeit in reverse, by making events look more gradual than they truly were (i.e., the Jaanusson effect or Sppil-Rongis effect; Jaanusson 1976; Marshall 1995b, 1998). However, sampling induces the opposite effect on the expected frequencies of sampled divergences (= nodes on a cladogram) during radiations, as we expect the frequency of nodes to increase before $\lambda$ increases (Figs. 8B, 9B). As a corollary, the expected branch durations/stratigraphic gaps (E[d]; Figs. 10B, 11B) should decrease before $\lambda$ increases.

The blurring effect becomes more pronounced as $\psi$ decreases. To envision why this would be, suppose that we are examining two Ordovician echinoderm and brachiopod clades that show elevated net diversification during the Darriwilian. The Jaanusson/Sppil-Rongis effect should smear the first appearances of taxa appearing during the radiation toward later Ordovician stages (e.g., the Sandbian or even Katian). This should be more true for echinoderms than for brachiopods, because pertaxon sampling rates generally are higher for brachiopods than for echinoderms (Foote and Sepkoski 1999). However, unsampled Dapingian ancestral species will have higher probabilities of generating sampled descendants than will unsampled Floian ancestral species: Dapingian species have greater probabilities than do Floian species of having successors that radiate in the Darriwilian. The effect also should be more dramatic for echinoderms than for brachiopods. Dapingian strata should yield more brachiopod species than echinoderm species with Darriwilian descendants (or that had near relatives with Darriwilian descendants). In other words, we should be sampling more of both the "dark gray/dark blue" and "light gray/yellow" lineages from Figure 1 for Dapingian brachiopods than for Dapingian echinoderms. As a corollary, compared with brachiopods, echinoderms should have more Dapingian nodes that link two Darriwilian nodes rather than one or more sampled taxa. This results in reduced branch durations and individual stratigraphic gaps per branch while at the same time permitting the Jaanusson/ Sppil-Rongis effect with sampled taxa.

An additional corollary to the argument above is that elevated origination rates following elevated extinction rates will partially offset the effects of extinction on expected branch durations and nodes within a tree (Figs. 8E11E). Thus, simple summaries of how branch durations and/or nodes from phylogenies are distributed over time might miss major turnovers. Instead, we need to compare the distributions of branch durations/stratigraphic gaps: relative to other intervals, species and branches dated to the rebound interval should show a greater proportion of both relatively short and relatively long branches compared with "normal" intervals with the same expected branch durations. Basically, we should either have a short branch duration confined to the rebound interval or a much longer one stretching back well into the extinction interval or even earlier. Alternatively, distributions of these parameters from particular portions of phylogenies (e.g., Soul and Friedman 2017) or simply "traditional" quantitative methods such as paleobiologists have been developing for decades will be of more use here.

Implications for Morphological Rate Studies and Tip-Dating

It is common for morphological disparity to peak early in clade histories (Hughes et al. 2013). Tip-dating approaches should be prone to implying deep divergence times for such clades to account for the large numbers of differences among early taxa. However, if those early bursts of disparity accompany elevated 
rates of diversification, then deep divergence times will be improbable given birth-deathsampling models. This could add considerable power to tree-based studies of shifting rates of morphological change and offer an improvement on prior assessments of the "deep unsampled divergences" alternative compared with sampling alone (e.g., Wagner 1995b; Ruta et al. 2006; Hopkins and Smith 2015). Moreover, appropriate priors on branch durations should at least somewhat mitigate the effects of correlated character change, which likely is common among fossil taxa (Wagner and Estabrook 2015), and which should mislead tipdating methods by implying numerous independent changes when only a single change altering multiple characters occurred (see, e.g., Pagel and Meade 2006; Beaulieu and Donoghue 2013; Herrera and Dávalos 2016).

Implications for Analyses of Stratigraphic Gaps within Phylogenies

As noted earlier, the branch durations implicit to phylogenies correspond to gaps in stratigraphic sampling within clades. Many studies contrast the stratigraphic gaps implicit to inferred phylogenies from different taxa or intervals of time (e.g., Benton and Storrs 1994; Hitchin and Benton 1997; Wills 1999, 2007; Benton et al. 2000; O'Connor et al. 2011). O'Connor and Wills (2016) assess a variety of reasons why differences might exist other than sampling, although they do not include differences in diversification rates. Bapst (2013) shows that differences in diversification offer another reason why two clades with otherwise similar preservation potential will have phylogenies with different summed branch durations and thus stratigraphic gaps. To this, we need to add heterogeneity in diversification parameters as a reason why two clades with similar preservation potential and similar general diversification dynamics should have different summed stratigraphic gaps.

\section{Accommodating Pulsed Turnover}

The estimates of $\Phi$ and expected branch duration probabilities presented earlier assume that origination and extinction are always happening. Pulsed turnover models posit that origination and/or extinction might be concentrated at the ends/onsets of what we now recognize as stratigraphic boundaries (Foote 2005). Purely pulsed extinction with continuous origination posits pure birth for diversification within an interval. Now the probability of one species having $K-1$ descendants after duration $t$ is:

$$
P[K \mid \lambda, t]=e^{-\lambda t} \times\left(1-e^{-\lambda t}\right)^{K-1}
$$

(Yule 1925). Equations (2-5) still give the probabilities of $0 \ldots N$ species surviving given some extinction rate. Note that $\Phi$ will be much higher at any given combination of $\lambda, \mu$, and $\psi$ given pulsed extinction than given continuous extinction, because $\mathrm{P}[0$ species $]=0$ until the very end of the interval. Thus, an interval of length $T$ has a minimum LMyr $=T$ rather than nearly zero. For pulsed origination, pure birth can be used for diversification at the outset of the interval. If both birth and death are pulsed, then $\Phi$ is maximized, because all lineages will persist for (essentially) the entire interval. This is essentially a corollary of Foote's (2005) demonstration that pulsed origination and extinction increase the proportion of taxa that we should first/last observe in the first/ last intervals at any given $\psi$. Of course, pulsed origination also hugely simplifies searching for divergence times: all branching would be concentrated in a small number of short intervals under a pure turnover-pulse model.

Finally, we might have mixed models in which there is some background rate and then some short-term pulsed rate. This was illustrated earlier under the pulsed extinction models (Figs. 8D,E-11D,E). To some extent, using time-slices finer than intervals, as done here, obviates the entire issue: the pure pulsed models then would have only the first and/or last time-slices with rates above zero.

Accommodating Within-Interval Variation in Diversification and Sampling among Clade Members

We can accommodate rate variation among taxa within time intervals by using hyperpriors on the rate distributions (see Heath 2012). For example, sampling distributions among contemporaneous taxa often fit log-normal distributions (Wagner and Marcot 2013; Foote 
2016). One could use the rates from midpoints of the four quartiles and calculate the average $\Phi$, the average probability of a sampled divergence, and the average probability of 0 finds given those four rates (see, e.g., Yang 1994). It is less clear what the appropriate distributions should be for $\lambda$ and $\mu$. Although paleontologists have modeled variation in extinction among intervals (e.g., Raup 1991; Wang 2003), we have not yet modeled variation in diversification among taxa within intervals. Heath et al. (2017) use exponential distributions for diversification hyperpriors. However, gamma distributions might be more appropriate than exponentials, as continuous origination and extinction rates are Poisson processes, and the gamma distribution is the conjugate prior for Poisson and related processes. Pulsed extinction is consistent with a binomial process (i.e., some proportion of species survive), and the conjugate prior there is a beta distribution. Yet another possibility is that $\lambda$ and $\mu$ reflect occupancy patterns within species and thus either follow or are affected by distributions describing occupancy such as log-normals (Foote 2016). This latter possibility also means that $\lambda$ and $\mu$ should covary among closely related species (see, e.g., Roy et al. 2009). If so, then skyline models allowing for heritable shifts in diversification rates might be most appropriate (Stadler et al. 2013; see also Stadler et al. 2018). Regardless, a very important question for paleobiologists to answer in the near future is: Exactly what distributions do best describe among-taxon variation in $\lambda$ and $\mu$ ?

\section{Other Issues}

Stadler et al. (2018) note that anagenetic transformation of one morphospecies to another, either during bifurcating cladogenesis (Fig. 1C) or within evolutionary lineages, will cause overestimates of origination and extinction implied by the stratigraphic ranges of named species. This in turn will alter our ideas on the probability of sampled sister taxa diverging. Many paleobiological studies suggest that anagenetic transformation on phylogenies is uncommon, but not nonexistent (Wagner and Erwin 1995; Polly 1997; Pardo et al. 2008; Bapst and Hopkins 2017). Stadler et al. (2018) suggest using two parameters, $\lambda_{B}$ and $\lambda_{\mathrm{A}}$, for branching and anagenetic speciation, respectively. One then could then modify either parameter $\lambda_{\mathrm{A}}$ over the course of Markov chain Monte Carlo searches, constraining $\lambda_{\mathrm{B} \cdot \mathrm{i}}+\lambda_{\mathrm{A} \cdot \mathrm{i}}=\lambda_{\mathrm{i}}$. Correspondingly, we would need to separate "true" extinction $\left(\mu_{\mathrm{T}}\right)$ from pseudoextinction rate (also $\lambda_{\mathrm{A} \cdot \mathrm{i}}$ ), so that $\mu_{\mathrm{T} \cdot \mathrm{i}}+\lambda_{\mathrm{A} \cdot \mathrm{i}}=\mu_{\mathrm{i}}$. Under this approach, if the trees maximizing the likelihood of morphological change models also imply copious anagenetic change, then those trees will not have reduced priors due to not having the sampled sister taxa predicted by $\lambda$ (or $\lambda_{1 \ldots N}$ for $N$ intervals), as they instead will use the lower $\lambda_{\mathrm{B}}$. and higher $\lambda_{\mathrm{A}}$.

\section{Conclusion}

As Bapst (2013) stresses, the probabilities of branch durations and divergence times represent a three-rate problem, with diversification and sampling affecting expected branch durations and stratigraphic gaps within phylogenies of fossil taxa. Heterogeneity in diversification over time also affects these expectations. The method presented herein allows paleobiologists to accommodate temporal variation in diversification and sampling that so many paleobiological studies document, and potentially overcomes a chief criticism of birth-death-sampling models (e.g., Marshall 2017). Given that current methods for modeling diversification and sampling with fossil data are almost certainly more advanced than are our current methods for modeling anatomical character evolution, the approach developed here provides paleobiologists with a much-needed independent test of divergence times implicit to alternate phylogenetic hypotheses, and thus an additional control when testing ideas about relationships or patterns of evolution across phylogeny.

\section{Acknowledgments}

I thank R. Warnock for copious discussion about birth-death-sampling models in all of their different guises. D. Bapst, M. Foote, W. Kiessling, M. Sakamoto, and an anonymous reviewer provided very helpful comments. For Katian gastropod data entered into the Paleobiology Database, I thank S. Holland and B. Kröger. This research was initiated under National 
Science Foundation grant EAR-0207874. This is Paleobiology Database Publication \#319.

\section{Literature Cited}

Alroy, J. 2014. Accurate and precise estimates of origination and extinction rates. Paleobiology 374-397.

Bapst, D. W. 2013. A stochastic rate-calibrated method for timescaling phylogenies of fossil taxa. Methods in Ecology and Evolution 4:724-733.

- 2016. paleotree. Program distributed by https://github.com/ dwbapst/paleotree.

Bapst, D. W., and M. J. Hopkins. 2017. Comparing cal3 and other a posteriori time-scaling approaches in a case study with the pterocephaliid trilobites. Paleobiology 43:49-67.

Bapst, D. W., A. M. Wright, N. J. Matzke, and G. T. Lloyd. 2016. Topology, divergence dates, and macroevolutionary inferences vary between different tip-dating approaches applied to fossil theropods (Dinosauria). Biology Letters 12:20160237.

Beaulieu, J. M., and M. J. Donoghue. 2013. Fruit evolution and diversification in campanulid angiosperms. Evolution 67:3132-3144.

Benson, R. B. J., G. Hunt, M. T. Carrano, and N. Campione. 2018. Cope's rule and the adaptive landscape of dinosaur body size evolution. Palaeontology 61:13-48.

Benton, M. J., and G. W. Storrs. 1994. Testing the quality of the fossil record: paleontological knowledge is improving. Geology 22:111-114.

Benton, M. J., M. A. Wills, and R. Hitchin. 2000. Quality of the fossil record through time. Nature 403:534-537.

Bergström, S. M., X. Chen, J. C. Gutiérrez-Marco, and A. Dronov. 2009. The new chronostratigraphic classification of the Ordovician System and its relations to major regional series and stages and to $\delta^{13} \mathrm{C}$ chemostratigraphy. Lethaia 42:97-107.

Connolly, S. R., and A. I. Miller. 2001. Joint estimation of sampling and turnover rates from fossil databases: capture-mark-recapture methods revisited. Paleobiology 27:751-767.

Didier, G., M. Fau, and M. Laurin. 2017. Likelihood of tree topologies with fossils and diversification rate estimation. Systematic Biology 66:964-987.

Finarelli, J. A., and L. H. Liow. 2016. Diversification histories for North American and Eurasian carnivorans. Biological Journal of the Linnean Society 118:26-38.

Fisher, D. C. 1994. Stratocladistics: morphological and temporal patterns and their relation to phylogenetic process. Pp. 133-171 in L. Grande and O. Rieppel, eds. Interpreting the hierarchy of nature - from systematic patterns to evolutionary process theories. Academic Press, Orlando, Fla.

Foote, M. 1996. On the probability of ancestors in the fossil record. Paleobiology 22:141-151.

1997. Estimating taxonomic durations and preservation probability. Paleobiology 23:278-300.

- 2001. Inferring temporal patterns of preservation, origination, and extinction from taxonomic survivorship analysis. Paleobiology 27:602-630.

- 2003. Origination and extinction through the Phanerozoic: a new approach. Journal of Geology 111:125-148.

- 2005. Pulsed origination and extinction in the marine realm. Paleobiology 31:6-20.

- 2016. On the measurement of occupancy in ecology and paleontology. Paleobiology 42:707-729.

Foote, M., and J. J. Sepkoski Jr. 1999. Absolute measures of the completeness of the fossil record. Nature 398:415-417.

Foote, M., J. P. Hunter, C. M. Janis, and J. J. Sepkoski Jr. 1999. Evolutionary and preservational constraints on origins of biologic groups: divergence times of eutherian mammals. Science 283:1310-1314.
Foote, M., R. A. Cooper, J. S. Crampton, and P. M. Sadler. 2018. Diversity-dependent evolutionary rates in early Palaeozoic zooplankton. Proceedings of the Royal Society of London B 285:20180122.

Friedman, M. 2010. Explosive morphological diversification of spiny-finned teleost fishes in the aftermath of the end-Cretaceous extinction. Proceedings of the Royal Society of London B 277:1675-1683.

Gavryushkina, A., T. A. Heath, D. T. Ksepka, T. Stadler, D. Welch, and A. J. Drummond. 2016. Bayesian total-evidence dating reveals the Recent crown radiation of penguins. Systematic Biology 66:57-73.

Gradstein, F. M., J. G. Ogg, and M. Schmitz. 2012. The geologic time scale 2012. Elsevier, Amsterdam.

Halliday, T. J. D., and A. Goswami. 2016. The impact of phylogenetic dating method on interpreting trait evolution: a case study of Cretaceous-Palaeogene eutherian body-size evolution. Biology Letters 12:20160051.

Heath, T. A. 2012. A hierarchical Bayesian model for calibrating estimates of species divergence times. Systematic Biology 61:793-809.

Heath, T. A., J. P. Huelsenbeck, and T. Stadler. 2014. The fossilized birth-death process for coherent calibration of divergence-time estimates. Proceedings of the National Academy of Sciences USA 111:E2957-E2966.

Heath, T. A., A. M. Wright, and W. Pett. 2017. Combined-evidence analysis of fossil and extant taxa under the fossilized birth-death process. RevBayes tutorial in S. Höhna, M. J. Landis, and T. A. Heath, eds. Phylogenetic inference using RevBayes. Current Protocols in Bioinformatics, 57:6.16.1-6.16.34. https://revbayes. github.io/tutorials/fbd/fbd_specimen.html.

Herrera, J. P., and L. M. Dávalos. 2016. Phylogeny and divergence times of lemurs inferred with Recent and ancient fossils in the tree. Systematic Biology 65:772-791.

Hitchin, R., and M. J. Benton. 1997. Congruence between parsimony and stratigraphy: comparisons of tree indices. Paleobiology 23:20-32.

Hopkins, M. J., and A. B. Smith. 2015. Dynamic evolutionary change in post-Paleozoic echinoids and the importance of scale when interpreting changes in rates of evolution. Proceedings of the National Academy of Sciences USA 112:3758-3763.

Huelsenbeck, J. P., and B. Rannala. 1997. Maximum likelihood estimation of topology and node times using stratigraphic data. Paleobiology 23:174-180.

Hughes, M., S. Gerber, and M. A. Wills. 2013. Clades reach highest morphological disparity early in their evolution. Proceedings of the National Academy of Sciences USA 110:13875-13879.

Jaanusson, V. 1976. Faunal dynamics in the middle Ordovician (Viruan) of Balto-Scandia. Pp. 301-326 in M. G. Bassett, ed. The Ordovician System: proceedings of a palaeontological association symposium. University of Wales Press and National Museum of Wales, Cardiff.

Jin, Y. G., Y. Wang, W. Wang, Q. H. Shang, C. Q. Cao, and D. H. Erwin 2000. Pattern of marine mass extinction near the Permian-Triassic boundary in South China. Science 289:432-436.

Lewis, P. O. 2001. A likelihood approach to estimating phylogeny from discrete morphological character data. Systematic Biology 50:913-925.

Liow, L. H., and J. A. Finarelli. 2014. A dynamic global equilibrium in carnivoran diversification over 20 million years. Proceedings of the Royal Society of London B 281:20132312.

Liow, L. H., and J. D. Nichols. 2010. Estimating rates and probabilities of origination and extinction using taxonomic occurrence data: capture-mark-recapture (CMR) approaches. Pp. 81-94 in J. Alroy and G. Hunt, eds. Quantitative methods in paleobiology. Paleontological Society, New Haven, Conn.

Lu, P. J., M. Yogo, and C. R. Marshall. 2006. Phanerozoic marine biodiversity dynamics in light of the incompleteness of the fossil record. Proceedings of the National Academy of Sciences USA 103:2736-2739. 
Marshall, C. R. 1995a. Distinguishing between sudden and gradual extinctions in the fossil record: predicting the position of the iridium anomaly using the ammonite fossil record on Seymour Island, Antarctica. Geology 23:731-734.

- 1995b. Stratigraphy, the true order of species' originations and extinctions, and testing ancestor-descendant hypotheses among Caribbean bryozoans. Pp. 208-236 in D. H. Erwin and R. L. Anstey, eds. New approaches to studying speciation in the fossil record. Columbia University Press, New York.

1998. Determining stratigraphic ranges. Pp. 23-53 in C. R. C. Paul and S. K. Donovan, eds. The adequacy of the fossil record. Wiley, New York.

- 2017. Five palaeobiological laws needed to understand the evolution of the living biota. Nature Ecology and Evolution 1:0165.

Matzke, N. J., and R. B. Irmis. 2018. Including autapomorphies is important for paleontological tip-dating with clocklike data, but not with non-clock data. PeerJ 6:e4553.

Matzke, N. J., and A. M. Wright. 2016. Inferring node dates from tip dates in fossil Canidae: the importance of tree priors. Biology Letters 12:2016032.

Miller, A. I., and J. J. Sepkoski Jr. 1988. Modeling bivalve diversification: the effect of interaction on a macroevolutionary system. Paleobiology 14:364-369.

O'Connor, A., and M. A. Wills. 2016. Measuring stratigraphic congruence across trees, higher taxa, and time. Systematic Biology 65:792-811.

O'Connor, A., C. Moncrieff, and M. A. Wills. 2011. Variation in stratigraphic congruence (GER) through the Phanerozoic and across higher taxa is partially determined by sources of bias. Geological Society of London Special Publication 358:31-52.

Pagel, M. D., and A. Meade. 2006. Bayesian analysis of correlated evolution of discrete characters by reversible-jump Markov chain Monte Carlo. American Naturalist 167:808-825.

Pardo, J. D., A. K. Huttenlocker, and J. D. Marcot. 2008. Stratocladistics and evaluation of evolutionary modes in the fossil record: an example from the ammonite genus Semiformiceras. Palaeontology 51:767-773.

Peters, S. E., and M. Foote. 2001. Biodiversity in the Phanerozoic: a reinterpretation. Paleobiology 27:583-601.

Polly, P. D. 1997. Ancestry and species definition in paleontology: a stratocladistic analysis of Paleocene-Eocene Viverravidae (Mammali, Carnivora) from Wyoming. Contributions from the Museum of Paleontology, University of Michigan 30:1-53.

Raup, D. M. 1972. Taxonomic diversity during the Phanerozoic. Science 177:1065-1071.

_ 1979. Biases in the fossil record of species and genera. Bulletin of the Carnegie Museum of Natural History 13:85-91.

1985. Mathematical models of cladogenesis. Paleobiology 11:42-52.

_. 1986. Biological extinction in earth history. Science 231:1528 1533.

- 1991. A kill curve for Phanerozoic marine species. Paleobiology 17:37-48

Ronquist, F., S. Klopfstein, L. Vilhelmsen, S. Schulmeister, D. L. Murray, and A. P. Rasnitsyn. 2012. A total-evidence approach to dating with fossils, applied to the early radiation of the Hymenoptera. Systematic Biology 61:973-999.

Roy, K., G. Hunt, and D. Jablonski. 2009. Phylogenetic conservatism of extinctions in marine bivalves. Science 325:733-737.

Ruta, M., P. J. Wagner, and M. I. Coates. 2006. Evolutionary patterns in early tetrapods. I. Rapid initial diversification by decrease in rates of character change. Proceedings of the Royal Society of London B 273:2107-2111.

Sakamoto, M., M. J. Benton, and C. Venditti. 2016. Dinosaurs in decline tens of millions of years before their final extinction. Proceedings of the National Academy of Sciences USA 113:50365040.
Sansom, R. S., and M. A. Wills. 2017. Differences between hard and soft phylogenetic data. Proceedings of the Royal Society of London B 284:20172150.

Sheehan, P. M. 1977. Species diversity in the Phanerozoic: a reflection of labor by systematists? Paleobiology 3:325-328.

Signor, P. W., and J. H. Lipps. 1982. Sampling bias, gradual extinction patterns and catastrophes in the fossil record. Geological Society of America Special Paper 190:291-296.

Smith, A. B. 1988. Patterns of diversification and extinction in early Palaeozoic echinoderms. Palaeontology 31:799-828.

- 2001a. Large-scale heterogeneity of the fossil record: implications for Phanerozoic biodiversity studies. Philosophical Transactions of the Royal Society of London B 356:351-367.

- 2001b. Probing the cassiduloid origins of clypeasteroid echinoids using stratigraphically restricted parsimony analysis. Paleobiology 27:392-404.

Soul, L. C., and M. Friedman. 2017. Bias in phylogenetic measurements of extinction and a case study of end-Permian tetrapods. Palaeontology 60:169-185.

Stadler, T. 2010. Sampling-through-time in birth-death trees. Journal of Theoretical Biology 267:396-404.

Stadler, T., D. Kühnert, S. Bonhoeffer, and A. J. Drummond. 2013. Birth-death skyline plot reveals temporal changes of epidemic spread in HIV and hepatitis C virus (HCV). Proceedings of the National Academy of Sciences USA 110:228-233.

Stadler, T., A. Gavryushkina, R. C. M. Warnock, A. J. Drummond, and T. A. Heath. 2018. The fossilized birth-death model for the analysis of stratigraphic range data under different speciation concepts. Journal of Theoretical Biology 447:41-45.

Starrfelt, J., and L. H. Liow. 2016. How many dinosaur species were there? Fossil bias and true richness estimated using a Poisson sampling model. Philosophical Transactions of the Royal Society of London B 371:20150219.

Wagner, P. J. 1995a. Stratigraphic tests of cladistic hypotheses. Paleobiology 21:153-178.

_. 1995b. Testing evolutionary constraint hypotheses with early Paleozoic gastropods. Paleobiology 21:248-272.

- 2000a. Exhaustion of cladistic character states among fossil taxa. Evolution 54:365-386.

- 2000b. Likelihood tests of hypothesized durations: determining and accommodating biasing factors. Paleobiology 26:431449.

Wagner, P. J., and D. H. Erwin. 1995. Phylogenetic patterns as tests of speciation models. Pp. 87-122 in D. H. Erwin and R. L. Anstey, eds. New approaches to studying speciation in the fossil record. Columbia University Press, New York.

Wagner, P. J., and G. F. Estabrook. 2015. The implications of stratigraphic compatibility for character integration among fossil taxa. Systematic Biology 64:838-852.

Wagner, P. J., and J. D. Marcot. 2013. Modelling distributions of fossil sampling rates over time, space and taxa: assessment and implications for macroevolutionary studies. Methods in Ecology and Evolution 4:703-713.

Wang, S. C. 2003. On the continuity of background and mass extinction. Paleobiology 29:455-467.

Wang, S. C., and C. R. Marshall. 2004. Improved confidence intervals for estimating the position of a mass extinction boundary. Paleobiology 30:5-18.

Wang, Y., P. M. Sadler, S.-z Shen, D. H. Erwin, Y.-c Zhang, X.-d. Wang, W. Wang, J. L. Crowley, and C. M. Henderson. 2014. Quantifying the process and abruptness of the endPermian mass extinction. Paleobiology 40:113-129.

Wills, M. A. 1999. The congruence between phylogeny and stratigraphy: randomization tests and the gap excess ratio. Systematic Biology 48:559-580.

- 2007. Fossil ghost ranges are most common in some of the oldest and some of the youngest strata. Proceedings of the Royal Society of London B 274:2421-2427. 
Wright, A. M., G. T. Lloyd, and D. M. Hillis. 2016. Modeling character change heterogeneity in phylogenetic analyses of morphology through the use of priors. Systematic Biology 65:602-611.

Wright, D. F. 2017. Bayesian estimation of fossil phylogenies and the evolution of early to middle Paleozoic crinoids (Echinodermata). Journal of Paleontology 91:799-814.

Wright, D. F., and U. Toom. 2017. New crinoids from the Baltic region (Estonia): fossil tip-dating phylogenetics constrains the origin and Ordovician-Silurian diversification of the Flexibilia (Echinodermata). Palaeontology 60:893-910.

Yacobucci, M. M. 1999. Plasticity of developmental timing as the underlying cause of high speciation rates in ammonoids. An example from the Cenomanian Western Interior Seaway of North America. Pp. 59-77 in F. Olóriz and F. J. RodriguezTovar, eds. Advancing research on living and fossil cephalopods. Kluwer Academic/Plenum, New York.

Yang, Z. 1994. Maximum likelihood phylogenetic estimation from DNA sequences with variable rates over sites: approximate methods. Journal of Molecular Evolution 39:306-314.

Yule, G. U. 1925. A mathematical theory of evolution, based on the conclusions of Dr. J. C. Willis, F.R.S. Philosophical Transactions of the Royal Society of London B 213:21-87.

Zhang, C., T. Stadler, S. Klopfstein, T. A. Heath, and F. Ronquist. 2016. Total-evidence dating under the fossilized birth-death process. Systematic Biology 65:228-249. 